\title{
The Prime Function, the Fay Trisecant Identity, and the van der Pauw Method
}

\section{On Some Conjectures on the Resistivity of a Holey Conductor}

\author{
Hiroyuki Miyoshi ${ }^{1} \cdot$ Darren Crowdy $^{1} \cdot$ Rhodri Nelson $^{2}$
}

Received: 6 October 2020 / Accepted: 29 March 2021 / Published online: 31 August 2021

(c) The Author(s) 2021

\begin{abstract}
The van der Pauw method is a well-known experimental technique in the applied sciences for measuring physical quantities such as the electrical conductivity or the Hall coefficient of a given sample. Its popularity is attributable to its flexibility: the same method works for planar samples of any shape provided they are simply connected. Mathematically, the method is based on the cross-ratio identity. Much recent work has been done by applied scientists attempting to extend the van der Pauw method to samples with holes ("holey samples"). In this article we show the relevance of two new function theoretic ingredients to this area of application: the prime function associated with the Schottky double of a multiply connected planar domain and the Fay trisecant identity involving that prime function. We focus here on the single-hole (doubly connected, or genus one) case. Using these new theoretical ingredients we are able to prove several mathematical conjectures put forward in the applied science literature.
\end{abstract}

Keywords Cross ratio · Prime function · Fay trisecant identity $\cdot$ van der Pauw

This article is dedicated to our former colleague, Professor Walter Hayman FRS, for his constant support over the years and his unwavering interest in what function theory can do for applications. His stories, anecdotes and songs, his career, and his fascinating life, remain an inspiration to us all at Imperial College London.

Communicated by James K. Langley.

$凶$ Hiroyuki Miyoshi

hiroyuki.miyoshi17@imperial.ac.uk

1 Department of Mathematics, Imperial College London, 180 Queen's Gate, London SW7 2AZ, UK

2 Department of Earth Science and Engineering, Royal School of Mines, Prince Consort Rd, South Kensington, London SW7 2BP, UK 
Mathematics Subject Classification 30Cxx $\cdot 14 \mathrm{Qxx} \cdot 31 \mathrm{Axx}$

\section{Introduction}

One of the most prevalent and successful measurement techniques in the applied sciences is the four-point probe method [1,2]. The van der Pauw method [3,4] is an example of such a method used to measure the resistivity (or its inverse quantity, the conductivity) of a conducting laminate sample. Van der Pauw's name is associated to this method because he was the first to point out that, owing to the conformal invariance of the underlying boundary value problem for the electrical voltage potential, the basic idea of the four-point method works for determining the resistivity of uniform twodimensional samples of any shape provided the contacts are placed at the edges of the sample [3,4]. Samples must have a flat shape of unit thickness, be isotropic of uniform resistivity, and be simply connected. That is, they must not have holes.

Figure 1 shows a set-up for the original van der Pauw measurement: four electrical contacts $\left(\Omega_{a}, \Omega_{b}, \Omega_{z}, \Omega_{w}\right)$ are placed on the perimeter of a test sample. If $\Omega_{a}$ and $\Omega_{b}$ represent a point source and sink of current $J_{a b}$ respectively, then the potential difference $V_{z w}$ between two other points $\Omega_{z}$ and $\Omega_{w}$ can be measured while this current is flowing. The resistance $R_{a b}^{z w}=V_{z w} / J_{a b}$ is then a measured quantity; a second resistance $R_{a w}^{z b}$ can be measured in exactly the same way. Van der Pauw [4] showed that for any arrangement of four electrical contacts, and given these two resistance measurements $R_{a b}^{z w}$ and $R_{a w}^{z b}$, the specific resistivity $\sigma$ can be found by solving the non-linear equation

$$
\exp \left(-\frac{R_{a b}^{z w}}{\lambda}\right)+\exp \left(-\frac{R_{a w}^{z b}}{\lambda}\right)=1
$$

with

$$
\lambda \equiv \frac{\sigma}{\pi d}
$$

and where $d$ is the uniform thickness of the two-dimensional sample. We will refer to this as the classical van der Pauw equation and it provides the basis for the van der Pauw method, the aim of which is to determine $\lambda$ and, hence, the sample resistivity. Because this method needs only two resistance measurements, the method is widely applied for measuring the resistivity of superconductors or Hall coefficients of materials in laboratory experiments $[3,5]$. An efficient numerical method to determine $\lambda$ is discussed in [6].

While powerful, the van der Pauw technique is limited by its applicability only to simply connected samples. In reality, a given sample may well have holes, and these will affect the voltage measurements. Consequently, in recent years the van der Pauw method for samples with a single hole, or several holes, has been studied extensively [7-16]. It has been reported that the van der Pauw equation (1) is inaccurate in samples with several holes [17]. This is not surprising given that the formula (1) takes no account of the presence of those holes. 
(i)

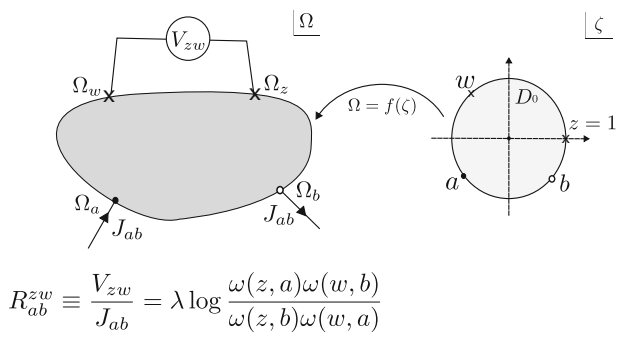

(ii)

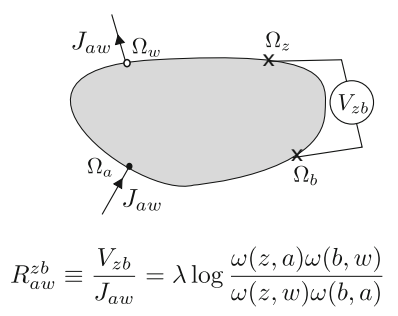

Fig. 1 The original van der Pauw set-up. The first measurement is the voltage difference between $z$ and $w$ with a point source of current at $a$ and a point sink of current at $b$. A second measurement is of the voltage difference between $z$ and $b$ with a source $a$ and a sink $w$. The associated resistances satisfy the van der Pauw equation (1) which can be solved for the sought-after parameter $\lambda$

The present paper has been inspired by several conjectures in the literature that have arisen in the aforementioned attempts to generalize the van der Pauw methodology to holey samples. Given that the original van der Pauw method rests on the cross-ratio identity, these conjectures represent an interesting challenge for function theorists. As we show here, two new mathematical tools turn out to be relevant to the van der Pauw problem as it pertains to holey samples: the theory of the so-called prime function on the Schottky double of a multiply connected planar domain [18] and the Fay trisecant identities also associated with those same compact Riemann surfaces and which involve the prime function. This article focusses on the function theory aspects. A companion article [19] explores some of the practical implications of our results.

In this paper, we use these mathematical tools - both of which are new to this application area-to prove two recent conjectures made in the applied science literature $[7,12]$. These conjectures concern a sample with an isolated hole, which is the natural first case to study. We now outline the nature of these conjectures.

It is a well-known consequence of an extension of the Riemann mapping theorem due to Koebe (see [20]) that any 2D sample with a single isolated hole can be transplanted conformally into an annulus $\rho<|\zeta|<1$ in a complex $\zeta$ plane, say, where the radius of the inner circle of the annulus depends on the shape of sample [18,21]. By conducting both numerical and actual experiments Szymański et al. showed [7] that the van der Pauw equation (1) does not hold for a sample with a hole but they conjectured that the data instead satisfies the inequality

$$
\exp \left(-\frac{R_{a b}^{z w}}{\lambda}\right)+\exp \left(-\frac{R_{a w}^{z b}}{\lambda}\right) \leq 1
$$

The same inequality has been mentioned in a series of papers [8,9]. It is one of the objectives of this paper to prove that (3) does indeed hold.

In [8] Szymański et al. also find that the pair of measured resistances $\left(R_{a b}^{z w}, R_{a w}^{z b}\right)$ satisfies another inequality which they dubbed a "lower envelope" and they proposed a method to measure $\lambda$ based on the existence of this envelope. To explain this, Fig. 2 shows the results of a repeat of the same numerical experiment conducted in [8]. It 
(i)
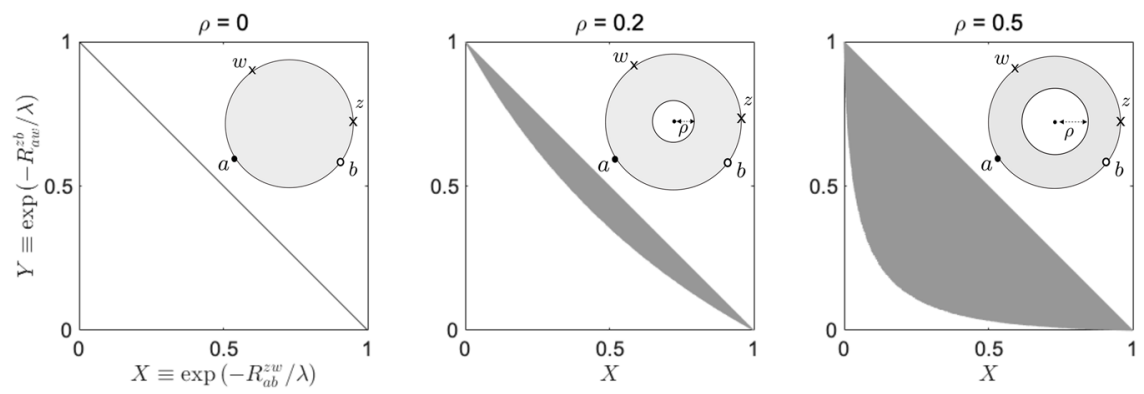

(ii)

Symmetric points with parameter $\theta$
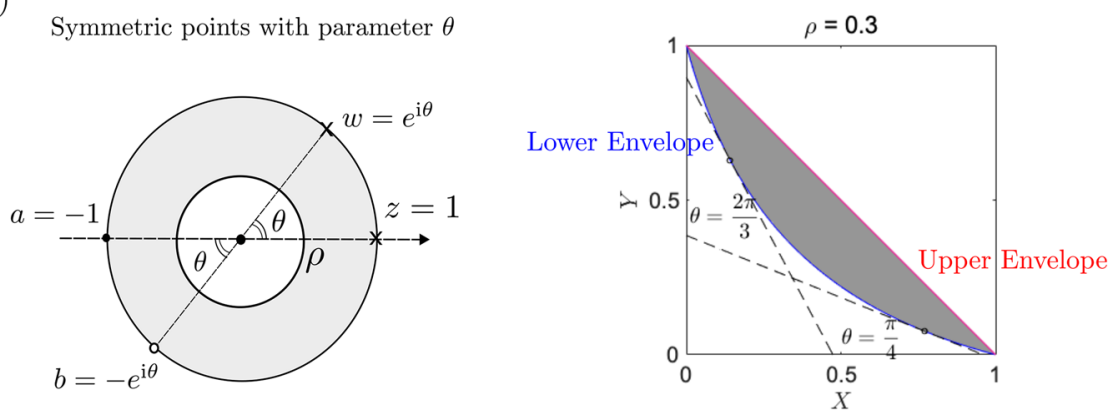

Fig. 2 (i) Evidence for the two "envelopes". Numerical experiments for pairs $(X, Y)$ where $X \equiv$ $\exp \left(-R_{a b}^{z w} / \lambda\right)$ and $Y \equiv \exp \left(-R_{a w}^{z b} / \lambda\right)$ following [8]. The point $z=1$ is fixed, but $(a, b, w)$ are picked at random with the ordering $0=\arg [z]<\arg [w]<\arg [a]<\arg [b]<2 \pi$. When $\rho=0$, all pairs $(X, Y)$ are on the line $X+Y=1$ which is (1). However, when $\rho>0$, all points $(X, Y)$ lie in the gray-shaded region bounded by the straight line $X+Y=1$ and a "lower envelope" which is curved. As shown in the center and right, the size of the gray-shaded area increases with $\rho$. (ii) The upper envelope (red line) and the lower envelope (blue line). The envelope is defined by its set of tangents. The gray region is above the tangent line for each point on the lower envelope. The figure to the left shows what we mean by the "symmetric" choice of contact points in which the sector formed by the pair $(a, b)$ subtends the same angle $\theta$ at the origin as that formed by the pair $(z, w)$

shows the data from 40,000 pairs $(X, Y)$, where

$$
X \equiv \exp \left(-R_{a b}^{z w} / \lambda\right), \quad Y \equiv \exp \left(-R_{a w}^{z b} / \lambda\right) .
$$

Three different samples are used with $\rho=0,0.2$ and 0.5. The contact points $z, w, a$, and $b$ are chosen at random but always such that they retain the ordering $0=\arg [z]<$ $\arg [w]<\arg [a]<\arg [b]<2 \pi$. The data is found to fall in the gray-shaded regions shown in Fig. 2. With no hole, which means $\rho=0$, the pair satisfies $X+Y=1$ which is just the original van der Pauw equation (1) in evidence. However, if $\rho>0$, this is no longer true and the data $(X, Y)$ "fills in" a crescent-shaped domain shown shaded in Fig. 2. Szymański et al. [8] conjecture that the data $(X, Y)$ always lies in such a domain bounded by the upper envelope $X+Y \leq 1$ and some lower envelope which depends only on the conformal modulus $\rho$. They also conjecture, again without a rigorous mathematical proof, that the lower envelope might correspond to the pair of 
$\left(X_{\theta}, Y_{\theta}\right)$, where $\left(X_{\theta}, Y_{\theta}\right)$ are measurements with the four electrical contacts having a certain symmetry shown on the lower left of Fig. 2(ii). The angle $\theta$ is defined as $\theta \equiv \arg [w / z]=\arg [b / a]$. In such a symmetric configuration the sector formed by the pair $(a, b)$ subtends the same angle $\theta$ at the origin as that formed by the pair $(z, w)$.

If these conjectures hold then the form of the upper and lower envelopes can be expressed mathematically as

$$
X+Y \leq 1, \quad Y-Y_{\theta} \geq \frac{\partial Y_{\theta} / \partial \theta}{\partial X_{\theta} / \partial \theta}\left(X-X_{\theta}\right), \quad \text { for } 0<\theta<\pi
$$

Two examples of the tangent line (5) on the lower envelope are shown on the right of Fig. 2(ii). These inequalities have been put forward in several papers [7-10], but no rigorous proof of them has yet been obtained.

This paper aims to understand the envelope structure mathematically and to offer proofs of the aforementioned conjectures. This is done by introducing, for the first time, two important tools into this area of investigation: (i) use of the prime function, denoted by $\omega(\zeta, c)$, associated with the concentric annulus $\rho<|\zeta|<1$; (ii) use of the Fay trisecant identity satisfied by this prime function. Using these tools we can gain insights into the two envelopes associated with the resistance measurements (4).

\section{The Van der Pauw Equation and the Cross Ratio Identity}

While applied scientists are familiar with the van der Pauw equation (1) mathematicians are perhaps more familiar with a similar-looking cross-ratio identity given by

$$
p_{0}(z, w ; b, a)+p_{0}(z, b ; w, a)=1
$$

where the classical cross-ratio is defined by

$$
p_{0}(z, w ; a, b) \equiv \frac{\omega(z, a) \omega(w, b)}{\omega(z, b) \omega(w, a)},
$$

and where, in a step usually not carried out, we introduce the prime function associated with the unit disc [18] as the simple monomial function

$$
\omega(\zeta, c)=\zeta-c
$$

Establishing the identity (6) is a simple exercise. In complex analysis the cross-ratio $[18,22]$ is most commonly encountered in a geometrical context as the Möbius mapping that provides a conformal mapping, as a function of the variable $z$, between 3 arbitrary complex points $(a, w, b)$ in the complex $z$ plane and the canonical choice of points $(0,1, \infty)$.

Concerning the prime function (8), it is so simple in this case that it is usually not even given the designation "prime function". However, a recent monograph [18] makes the case that recognizing it as the simplest instance of a more general notion of a prime 
function is important for generalizing many known results for simply connected planar geometries to multiply connected cases in a natural way. The van der Pauw problem of interest here is no exception: it will be shown later that the natural way to extend the classical van der Pauw method to multiply connected geometries is to treat the problem using the prime function - that is, the multiply connected generalization of (8) - and to make use of some important identities satisfied by those functions.

There is a connection between (1) and (6). It arises by considering the complex potential $H_{0}(\Omega)$ of the complex variable $\Omega=x+\mathrm{i} y$, where $(x, y)$ denotes Cartesian coordinates in the physical plane, whose real part is the harmonic voltage potential $V(x, y)$ in the sample:

$$
H_{0}(\Omega)=V(x, y)+\mathrm{i} \chi(x, y),
$$

where we have introduced $\chi(x, y)$, the harmonic conjugate of $V(x, y)$ in the physical $(x, y)$ plane. $V(x, y)$ is harmonic in the sample and its normal derivative vanishes on the sample boundary; equivalently, by the Cauchy-Riemann equations, the harmonic conjugate $\chi(x, y)$ is constant on the boundary.

By the well-known Riemann mapping theorem, and the conformal invariance of the boundary value problem for the potential $V(x, y)$ driven by a source at $\Omega_{a}$ and a compensating sink at $\Omega_{b}$ [22], the complex potential $h_{0}(\zeta) \equiv H_{0}(\Omega)$ is given by the explicit formula

$$
h_{0}(\zeta)=\frac{\sigma J_{a b}}{\pi d} \log \left(\frac{\omega(\zeta, a)}{\omega(\zeta, b)}\right)
$$

where, once again, we notice the appearance of the prime function (8) and

$$
\Omega_{a}=f(a), \quad \Omega_{b}=f(b),
$$

where $\Omega=f(\zeta)$ is the conformal mapping between the unit disc $D_{0}$ in the $\zeta$ plane and the given laminate sample in the complex $\Omega$ plane. The potential difference $V_{z w}$ is therefore given by

$$
\begin{aligned}
V_{z w} \equiv \operatorname{Re}\left[h_{0}(z)\right]-\operatorname{Re}\left[h_{0}(w)\right] & =\lambda J_{a b} \log \left|\frac{\omega(z, a) \omega(w, b)}{\omega(z, b) \omega(w, a)}\right| \\
& =\lambda J_{a b} \log \left|p_{0}(z, w ; a, b)\right|
\end{aligned}
$$

where $z$ and $w$ are the preimages of the two measurement contact points $\Omega_{z}$ and $\Omega_{w}$ :

$$
\Omega_{z}=f(z), \quad \Omega_{w}=f(w) .
$$

It follows that

$$
R_{a b}^{z w} \equiv \frac{V_{z w}}{J_{a b}}=\lambda \log p_{0}(z, w ; a, b),
$$


where we have removed the modulus symbols because the cross-ratio is real and positive when all the points $z, w, a$, and $b$ are on the unit circle in the $\zeta$ plane, and $0 \leq \arg [z]<\arg [w]<\arg [a]<\arg [b]<2 \pi$. On combining (14) with (6), we arrive at the van der Pauw equation (1).

\section{The Prime Function for the Annulus $\rho<|\zeta|<1$}

Now consider the van der Pauw method for a doubly connected sample. Let $D$ denote a bounded sample with an isolated hole. Let $\partial D_{0}$ be the outer boundary of the sample and $\partial D_{1}$ the boundary of the hole. Similar to the original van der Pauw method, we assume that the sample thickness is $d$. We assume that the hole in the sample carries no net charge. The set-up is illustrated in Fig. 3.

Suppose that 4 point contacts $\left(\Omega_{a}, \Omega_{b}, \Omega_{z}, \Omega_{w}\right)$, of infinitesimal width, are placed on $\partial D_{0}$. It is known, by an extension of the Riemann mapping theorem $[18,20]$, that any such domain is conformally equivalent to a concentric annulus $\rho<|\zeta|<1$ with circular boundaries $C_{0}$ and $C_{1}$ and $0 \leq \rho<1$. The circle $C_{0}$ is the unit circle; $C_{1}$ is the circle $|\zeta|=\rho$. In other words, there exists an analytic function

$$
\Omega=f(\zeta)
$$

that transplants the annulus $\rho<|\zeta|<1$ to the domain $D$ with $C_{0}$ being transplanted to $\partial D_{0}$ and $C_{1}$ to $\partial D_{1}$.

Let the required complex potential, as a function of $\Omega=x+\mathrm{i} y$, be

$$
H(\Omega)=V(x, y)+\mathrm{i} \chi(x, y) .
$$

We can still exploit the conformal invariance for the problem of determining the potential $V(x, y)$ in this doubly connected domain. Crowdy [18,23] has shown that the complex potentials for any point source/sink driven harmonic field in a multiply connected domain can be written down explicitly in terms of the prime function associated with that domain. It is important to emphasize, for possible future generalization of this work, that this fact holds for domains of any finite connectivity not just the doubly connected case of interest here.

The prime function $\omega(\zeta, c)$ for the annulus $\rho<|\zeta|<1$ can be defined explicitly in this case by the formula [18]

$$
\omega(\zeta, c)=-\frac{c}{\hat{P}(1)} P\left(\frac{\zeta}{c}\right)
$$

where

$$
P(\zeta) \equiv(1-\zeta) \hat{P}(\zeta), \quad \hat{P}(\zeta) \equiv \prod_{n=1}^{\infty}\left(1-\rho^{2 n} \zeta\right)\left(1-\frac{\rho^{2 n}}{\zeta}\right)
$$


(i)

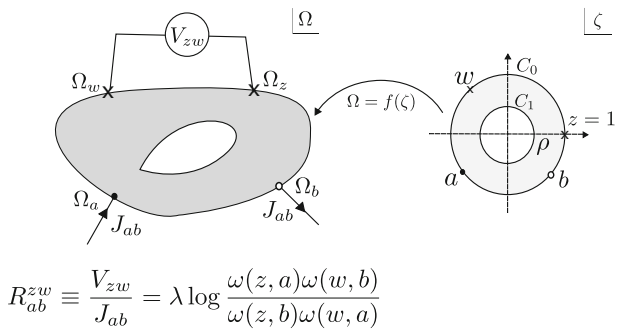

(ii)

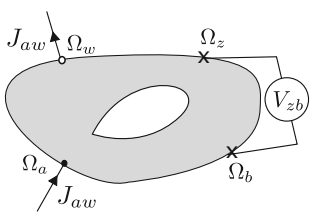

$R_{a w}^{z b} \equiv \frac{V_{z b}}{J_{a w}}=\lambda \log \frac{\omega(z, a) \omega(b, w)}{\omega(z, w) \omega(b, a)}$

Fig. 3 The van der Pauw set-up for a sample with an isolated hole. The first measurement is the voltage difference between $z$ and $w$ with a source of current $a$ and a sink of current $b$. The second measurement is the difference between $z$ and $b$ with a source $a$ and a sink $w$. Similar to the original van der Pauw method, the measured resistances are denoted by the logarithm of the Schottky-Klein prime function

It is easy to show, directly from these definitions via an infinite product, that

$$
P\left(\zeta^{-1}\right)=-\zeta^{-1} P(\zeta), \quad P\left(\rho^{2} \zeta\right)=-\zeta^{-1} P(\zeta)
$$

These identities, which are the fundamental properties of the prime function in this case (see [18, Ch. 5] which is devoted to this function and its properties), will be useful later. For notational brevity, we write $P(\zeta)$ even though this function also depends on the parameter $\rho$ as is clear from its definition (18). The reader should bear in mind this additional parametric dependence.

It is important to mention that while, merely for convenience and brevity of exposition, we have here defined the prime function for the concentric annulus in terms of an infinite product (18) the monograph [18] gives a more intrinsic derivation of the prime function for multiply connected planar domains using the first-type Green's function as the starting point. Interested readers are encouraged to refer there for a much more general mathematical perspective.

We will also need the functions $K(\zeta)$ and $L(\zeta)$ defined as

$$
K(\zeta) \equiv \zeta \frac{\partial}{\partial \zeta} \log P(\zeta), \quad L(\zeta) \equiv \zeta \frac{\partial K(\zeta)}{\partial \zeta}
$$

The logarithmic derivative of the prime function $K(\zeta)$ and its derivative $L(\zeta)$ are also important functions in the general function theory on multiply connected domains [18]. For the concentric annulus it is easily shown, on use of (19), that these functions satisfy the functional relations

$$
\begin{array}{cl}
K\left(\zeta^{-1}\right)=1-K(\zeta), & K\left(\rho^{2} \zeta\right)=K(\zeta)-1 \\
L\left(\zeta^{-1}\right)=L(\zeta), & L\left(\rho^{2} \zeta\right)=L(\zeta)
\end{array}
$$

Actually, the function $K(\zeta)$ can be related to the Weierstrass zeta function and $L(\zeta)$ to the Weierstrass $\wp$-function [18] but those theoretical connections will not be needed here and this remark is made merely as a point of interest. 
Following $[18,23]$ the complex potential $h(\zeta) \equiv H(f(\zeta))$ is given by

$$
h(\zeta)=\frac{\sigma J_{a b}}{2 \pi d} \log \left(\frac{\bar{a} b}{|a b|} \frac{\omega(\zeta, a) \omega\left(\zeta, \bar{a}^{-1}\right)}{\omega(\zeta, b) \omega\left(\zeta, \bar{b}^{-1}\right)}\right)=\lambda J_{a b} \log \left(\frac{a}{b} \frac{P(\zeta / a)}{P(\zeta / b)}\right)
$$

where the first equality is derived in $[18,23]$ and follows from the general properties of the prime function, and where we have used (17) in the second equality. The relations (11) and (13) give the relationship between $(a, b, z, w)$ and $\left(\Omega_{a}, \Omega_{b}, \Omega_{z}, \Omega_{w}\right)$ although it is understood that the mapping $f(\zeta)$ is now the new mapping from the concentric annulus to the holey sample. Note that, because two electrical contacts $a$ and $b$ are on $C_{0}, \bar{a}=a^{-1}$ and $\bar{b}=b^{-1}$.

The voltage difference between $z$ and $w$ is given by

$$
V_{a b}^{z w} \equiv \operatorname{Re}[h(z)]-\operatorname{Re}[h(w)]=\lambda J_{a b} \log \frac{P(z / a) P(w / b)}{P(z / b) P(w / a)} .
$$

Since all contacts are located on the same edge of the annulus, then $\operatorname{Im}[h(z)]-$ $\operatorname{Im}[h(w)]=0$. The measured resistance $R_{a b}^{z w}$ is defined as

$$
R_{a b}^{z w} \equiv \frac{V_{z w}}{J_{a b}}=\lambda \log \frac{P(z / a) P(w / b)}{P(z / b) P(w / a)}
$$

In the same way,

$$
R_{a w}^{z b} \equiv \frac{V_{z b}}{J_{a w}}=\lambda \log \frac{P(z / a) P(b / w)}{P(z / w) P(b / a)} .
$$

It is straightforward to check that the formulas (24) and (25) above are equivalent to those given in [7]. More precisely, it can be checked that the function $G(\phi)$ used in [7] is related to $P(\zeta)$ - and hence to the prime function for the concentric annulus (17) — by the formula

$$
P\left(e^{\mathrm{i} \phi}\right)=\left(1-e^{\mathrm{i} \phi}\right) \prod_{n=1}^{\infty}\left(1+\rho^{4 n}-2 \rho^{2 n} \cos \phi\right)=-2 \mathrm{i} e^{\mathrm{i} \phi / 2} \hat{P}(\mathrm{i}) G(\phi),
$$

where the function $G(\phi)$ introduced in [7] is defined by

$$
G(\phi) \equiv \sin \frac{\phi}{2} \prod_{n=1}^{\infty}\left(1-\frac{\cos \phi}{\cosh \tilde{h} n}\right), \quad \tilde{h} \equiv 2 \log \rho .
$$

Although (26) shows that our new expressions (24)-(25) coincide with those of [7], which is reassuring, there is much significance in having recognized that the resistances can be written in terms of this special transcendental function known as the prime function [18] of the preimage concentric annulus. 
First, the notion of a prime function extends to a planar domain of any finite connectivity [18] which means that we have already provided a route to generalizing all the ideas in this paper presented here for the annulus to any higher connected domain, that is, to a sample with more than one hole. Crowdy [23] was the first to show how the complex potentials for source/sink driven harmonic fields in multiply connected domains of arbitrary connectivity can be written explicitly in terms of the associated prime functions. His treatment uses irrotational fluid mechanics as the physical context but, mathematically, the problem is identical to the electrical conduction problems of interest here.

Second, it is known [18] that prime functions, including those associated with domains of connectivity higher than one, satisfy a so-called Fay trisecant identity. This identity can be viewed as an analogue of the cross-ratio identity (6) on a higher genus Riemann surface [24] and is the topic of the next section.

\section{The Fay Trisecant Identity for the Annulus $\rho<|\zeta|<1$}

It is useful to introduce the function

$$
p(z, w ; a, b) \equiv \frac{\omega(z, a) \omega(w, b)}{\omega(z, b) \omega(w, a)} .
$$

This formula is identical to that defining the cross-ratio (6) but this quantity is no longer a cross-ratio because the definition of the prime function has changed. On use of (17) formula (28) can be written in terms of $P(\zeta)$ as

$$
p(z, w ; a, b)=\frac{P(z / a) P(w / b)}{P(z / b) P(w / a)} .
$$

From (29) and (24)-(25) we see that

$$
\exp \left(-R_{a b}^{z w} / \lambda\right)=p(z, w ; b, a), \quad \exp \left(-R_{a w}^{z b} / \lambda\right)=p(z, b ; w, a)
$$

The Fay trisecant identity associated with this prime function is

$$
\frac{P(k z / w) P(k a / b)}{P(k z a / w b)} p(z, w ; b, a)+\frac{P(k z / b) P(k a / w)}{P(k z a / w b)} p(z, b ; w, a)=P(k),
$$

where $k$ is an arbitrary complex number. This statement (31) of the genus-1 Fay trisecant identity expressed purely in terms of the prime function of the concentric annulus has been taken from Exercise 8.9 of Chapter 8 of the monograph [18] which asks the reader to prove it using the properties of so-called loxodromic functions. While (31) is a particular form of the more general Fay trisecant identity [24,25] the authors have not found it written in the form (31) anywhere else in the literature (besides [18]). This form (31) of the genus-one Fay trisecant identity will be a crucial tool in what follows. 
Although it is well established [24,25] from more general arguments it is instructive to sketch a proof this form (31) of the genus-one Fay trisecant identity.

First we consider the left hand side of (31) as a function of $z$ with all other quantities being treated as parameters. Let

$$
J(z) \equiv \frac{P(k z / w) P(k a / b)}{P(k z a / w b)} p(z, w ; b, a)+\frac{P(k z / b) P(k a / w)}{P(k z a / w b)} p(z, b ; w, a) .
$$

The properties (19) of the function $P(\zeta)$ can be used to show that

$$
J\left(\rho^{2} z\right)=J(z) .
$$

Hence, since it is also meromorphic as a function of $z$, it is a loxodromic function of $z$; see [18, Ch. 8]. A loxodromic function is the name of an automorphic function on the Schottky double of the concentric annulus; it is a meromorphic function of that surface satisfying the functional identity (33). If we write

$$
J(z)=\frac{N(z)}{P(k z a / w b) P(z / a) P(w / b)}
$$

so that

$$
\begin{aligned}
N(z)= & P(k z / w) P(k a / b) P(z / b) P(w / a) \\
& -(w / b) P(k z / b) P(k a / w) P(z / w) P(b / a)
\end{aligned}
$$

then, it can be verified, again using the properties (19) of $P(\zeta)$, that

$$
N(a)=N(w b / k a)=0 .
$$

Since $J(z)$ is a loxodromic function with removable poles at $z=a$ and $z=w b / k a-$ and, therefore, having no poles on the surface - it must be independent of $z$, which means it is a constant when considered as a function of $z$. We are employing a Liouvilletype theorem on this genus-one Schottky double: any meromorphic function on it having no poles must be constant. Such results will be used extensively throughout this paper.

We can also consider the left hand side of (31) as a function for $w$ and write

$$
\tilde{J}(w) \equiv \frac{P(k z / w) P(k a / b)}{P(k z a / w b)} p(z, w ; b, a)+\frac{P(k z / b) P(k a / w)}{P(k z a / w b)} p(z, b ; w, a) .
$$

This can also be shown to be loxodromic, i.e.,

$$
\tilde{J}\left(\rho^{2} w\right)=\tilde{J}(w)
$$

and to have removable poles at $w=b$ and $w=k z a / b$. It is therefore independent of $w$. 
By similar arguments, considering the left hand side of (31) successively as a function of $a$ and $b$ it can be shown to be independent of those variables too. Putting all these facts together, we conclude that

$$
\frac{P(k z / w) P(k a / b)}{P(k z a / w b)} p(z, w ; b, a)+\frac{P(k z / b) P(k a / w)}{P(k z a / w b)} p(z, b ; w, a)=C(k),
$$

where $C(k)$ is a function to be determined. It can be found by matching to the limit of the left hand side of (39) in the double limit $w \rightarrow z$ and $b \rightarrow a$ which yields

$$
C(k)=P(k) .
$$

Thus we have established the Fay trisecant identity (31).

On substituting (24) and (25) into (31), we obtain

$$
\frac{P(k z / w) P(k a / b)}{P(k) P(k z a / w b)} \exp \left(-\frac{R_{a b}^{z w}}{\lambda}\right)+\frac{P(k z / b) P(k a / w)}{P(k) P(k z a / w b)} \exp \left(-\frac{R_{a w}^{z b}}{\lambda}\right)=1 .
$$

When $\rho \rightarrow 0$, so that there is no hole in a sample, it is straightforward to check that

$$
\frac{P(k z / w) P(k a / b)}{P(k) P(k z a / w b)}=\frac{P(k z / b) P(k a / w)}{P(k) P(k z a / w b)}=1
$$

because $P(\zeta)=1-\zeta$ when $\rho=0$. The original van der Pauw equation (1) is therefore retrieved from (41). All dependence on the new parameter $k$ disappears in this simply connected limit.

It is clear that (41) opens up new perspectives: that it reduces, as $\rho \rightarrow 0$, to the original van der Pauw equation (1) is tantalizing. It also makes it a natural candidate, at least from the mathematical point of view, to find ways to extend the van der Pauw method to holey samples. In contrast to the original van der Pauw equation, the coefficients of $\exp \left(-R_{a b}^{z w} / \lambda\right)$ and $\exp \left(-R_{a w}^{z b} / \lambda\right)$ in (41) now depend not only on the electrical contact locations $z, w, a, b$ but also on a fifth complex parameter $k$. It should be emphasized that (41) holds for arbitrary choices of $a, b, z, w$ and $k$ even though, for present purposes, we will assume that $a, b, z$ and $w$ lie on $C_{0}$. The freedom in the choice of $k$ will be exploited in the next section to gain insights into the envelope structure evident in Fig. 2.

\section{Analysis of the Envelopes: The Integrated Fay Identity}

In Sect. 1 we discussed the existence of two envelopes, an "upper" and a "lower" envelope with the mathematical definitions given in (5). It is now shown how the new tools introduced in the previous two sections allow us to prove the conjecture enshrined in (5). 


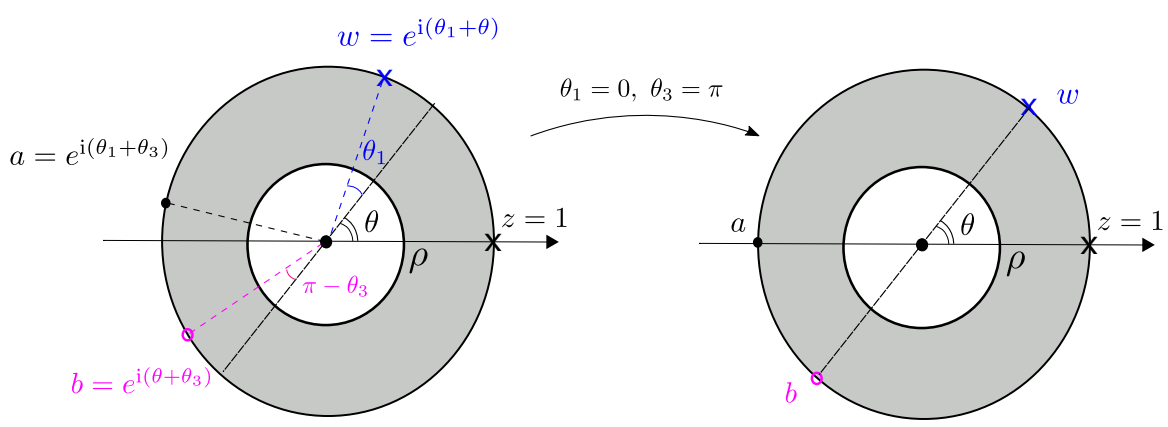

Fig. 4 Illustration of the complex coordinates. The parameters $\theta_{1}$ and $\theta_{3}$ are seen as displacements from the symmetric points where $z$ is fixed to 1

For arbitrary $z, w, a$, and $b$ on $C_{0}$ we can introduce new angular coordinates $\theta, \theta_{1}$ and $\theta_{3}$ :

$$
z=1, \quad w=\exp \left(\mathrm{i}\left(\theta_{1}+\theta\right)\right), \quad a=\exp \left(\mathrm{i}\left(\theta_{1}+\theta_{3}\right)\right), \quad b=\exp \left(\mathrm{i}\left(\theta+\theta_{3}\right)\right)
$$

Because $0<\arg [w]<\arg [a]<\arg [b]<2 \pi$, the range of $\theta_{1}, \theta, \theta_{3}$ are given by

$$
-\theta<\theta_{1}<\theta, \quad \theta<\theta_{3}<2 \pi-\theta, \quad 0<\theta<\pi .
$$

It is important to mention that the symmetry case shown in Fig. 2 corresponds to the choices $\theta_{1}=0$ and $\theta_{3}=\pi$. An illustration of the complex coordinates is shown in Fig. 4. The parameters $\theta_{1}$ and $\theta_{3}$ are seen as displacements from the symmetric points with parameter $\theta$.

The Fay trisecant identity (41) can be written

$$
A\left(\theta_{1}, \theta, \hat{k}\right) X_{\theta_{1}, \theta_{3}, \theta}+B\left(\theta_{3}, \theta, \hat{k}\right) Y_{\theta_{1}, \theta_{3}, \theta}=1,
$$

where

$$
\begin{aligned}
& X_{\theta_{1}, \theta_{3}, \theta} \equiv \exp \left(-\frac{R_{a b}^{z w}}{\lambda}\right)=\frac{P\left(e^{-\mathrm{i}\left(\theta+\theta_{3}\right)}\right) P\left(e^{\mathrm{i}\left(\theta-\theta_{3}\right)}\right)}{P\left(e^{-\mathrm{i}\left(\theta_{1}+\theta_{3}\right)}\right) P\left(e^{\mathrm{i}\left(\theta_{1}-\theta_{3}\right)}\right)}, \\
& Y_{\theta_{1}, \theta_{3}, \theta} \equiv \exp \left(-\frac{R_{a w}^{z b}}{\lambda}\right)=\frac{P\left(e^{-\mathrm{i}\left(\theta+\theta_{1}\right)}\right) P\left(e^{\mathrm{i}\left(\theta-\theta_{1}\right)}\right)}{P\left(e^{-\mathrm{i}\left(\theta_{1}+\theta_{3}\right)}\right) P\left(e^{\mathrm{i}\left(\theta_{3}-\theta_{1}\right)}\right)},
\end{aligned}
$$


and the coefficient functions are

$$
\begin{aligned}
A\left(\theta_{1}, \theta, \hat{k}\right) \equiv \frac{P(k z / w) P(k a / b)}{P(k) P(k z a / w b)} & =\frac{P\left(k e^{-\mathrm{i}\left(\theta+\theta_{1}\right)}\right) P\left(k e^{\mathrm{i}\left(-\theta+\theta_{1}\right)}\right)}{P(k) P\left(k e^{-2 \mathrm{i} \theta}\right)} \\
& =\frac{P\left(\hat{k} e^{-\mathrm{i} \theta_{1}}\right) P\left(\hat{k} e^{\mathrm{i} \theta_{1}}\right)}{P\left(\hat{k} e^{-\mathrm{i} \theta}\right) P\left(\hat{k} e^{\mathrm{i} \theta}\right)}, \\
B\left(\theta_{3}, \theta, \hat{k}\right) \equiv \frac{P(k z / b) P(k a / w)}{P(k) P(k z a / w b)} & =\frac{P\left(k e^{-\mathrm{i}\left(\theta+\theta_{3}\right)}\right) P\left(k e^{\mathrm{i}\left(-\theta+\theta_{3}\right)}\right)}{P(k) P\left(k e^{-2 \mathrm{i} \theta}\right)} \\
& =\frac{P\left(\hat{k} e^{-\mathrm{i} \theta_{3}}\right) P\left(\hat{k} e^{\mathrm{i} \theta_{3}}\right)}{P\left(\hat{k} e^{-\mathrm{i} \theta}\right) P\left(\hat{k} e^{\mathrm{i} \theta}\right)},
\end{aligned}
$$

and where we have set $k=\hat{k} e^{i \theta}$ because $k$ is arbitrary. Notice that, with this choice, the resistance measurements have the following symmetries:

$$
\begin{aligned}
X_{\theta_{1}, 2 \pi-\theta_{3}, \theta} & =X_{-\theta_{1}, \theta_{3}, \theta}=X_{-\theta_{1}, 2 \pi-\theta_{3}, \theta}=X_{\theta_{1}, \theta_{3}, \theta}, \\
Y_{\theta_{1}, 2 \pi-\theta_{3}, \theta} & =Y_{-\theta_{1}, \theta_{3}, \theta}=Y_{-\theta_{1}, 2 \pi-\theta_{3}, \theta}=Y_{\theta_{1}, \theta_{3}, \theta} .
\end{aligned}
$$

It is also important to note that $A\left(\theta_{1}, \theta, \hat{k}\right)$ is independent of $\theta_{3}$ and $B\left(\theta_{3}, \theta, \hat{k}\right)$ is independent of $\theta_{1}$.

A crucial next step is to consider an integrated form of the Fay trisecant identity (41). Consider integrals of $A\left(\theta_{1}, \theta, \hat{k}\right)$ and $B\left(\theta_{3}, \theta, \hat{k}\right)$ with respect to $\hat{k}$ around the circle $|\hat{k}|=\rho$. From (45), we obtain

$$
\alpha\left(\theta_{1}, \theta\right) X_{\theta_{1}, \theta_{3}, \theta}+\beta\left(\theta_{3}, \theta\right) Y_{\theta_{1}, \theta_{3}, \theta}=1,
$$

where

$$
\begin{aligned}
& \alpha\left(\theta_{1}, \theta\right) \equiv \frac{1}{2 \pi} \int_{0}^{2 \pi} A\left(\theta_{1}, \theta, \rho e^{\mathrm{i} \phi}\right) d \phi=\frac{1}{2 \pi} \int_{0}^{2 \pi} \frac{P\left(\rho e^{\mathrm{i}\left(\phi-\theta_{1}\right)}\right) P\left(\rho e^{\mathrm{i}\left(\phi+\theta_{1}\right)}\right)}{P\left(\rho e^{\mathrm{i}(\phi-\theta)}\right) P\left(\rho e^{\mathrm{i}(\phi+\theta)}\right)} d \phi, \\
& \beta\left(\theta_{3}, \theta\right) \equiv \frac{1}{2 \pi} \int_{0}^{2 \pi} B\left(\theta_{3}, \theta, \rho e^{\mathrm{i} \phi}\right) d \phi=\frac{1}{2 \pi} \int_{0}^{2 \pi} \frac{P\left(\rho e^{\mathrm{i}\left(\phi-\theta_{3}\right)}\right) P\left(\rho e^{\mathrm{i}\left(\phi+\theta_{3}\right)}\right)}{P\left(\rho e^{\mathrm{i}(\phi-\theta)}\right) P\left(\rho e^{\mathrm{i}(\phi+\theta)}\right)} d \phi .
\end{aligned}
$$

The integrated Fay's trisecant identity (49) turns out to be essential for proving the conjectures about the envelope structure. On taking a derivative of (49) with respect to $\theta$,

$$
\begin{aligned}
& \frac{\partial \alpha\left(\theta_{1}, \theta\right)}{\partial \theta} X_{\theta_{1}, \theta_{3}, \theta}+\frac{\partial \beta\left(\theta_{3}, \theta\right)}{\partial \theta} Y_{\theta_{1}, \theta_{3}, \theta} \\
& \quad+\alpha\left(\theta_{1}, \theta\right) \frac{\partial X_{\theta_{1}, \theta_{3}, \theta}}{\partial \theta}+\beta\left(\theta_{3}, \theta\right) \frac{\partial Y_{\theta_{1}, \theta_{3}, \theta}}{\partial \theta}=0 .
\end{aligned}
$$


The sum of the first two terms is zero because

$$
\begin{aligned}
& \frac{\partial \alpha\left(\theta_{1}, \theta\right)}{\partial \theta} X_{\theta_{1}, \theta_{3}, \theta}+\frac{\partial \beta\left(\theta_{3}, \theta\right)}{\partial \theta} Y_{\theta_{1}, \theta_{3}, \theta} \\
& =\frac{\mathrm{i}}{2 \pi} \int_{0}^{2 \pi}\left[A\left(\theta_{1}, \theta, \rho e^{\mathrm{i} \phi}\right) X_{\theta_{1}, \theta_{3}, \theta}+B\left(\theta_{3}, \theta, \rho e^{\mathrm{i} \phi}\right) Y_{\theta_{1}, \theta_{3}, \theta}\right] \\
& \quad \times\left[K\left(\rho e^{\mathrm{i}(\phi-\theta)}\right)-K\left(\rho e^{\mathrm{i}(\phi+\theta)}\right)\right] d \phi \\
& =\frac{\mathrm{i}}{2 \pi} \int_{0}^{2 \pi}\left[K\left(\rho e^{\mathrm{i}(\phi-\theta)}\right)-K\left(\rho e^{\mathrm{i}(\phi+\theta)}\right)\right] d \phi=0,
\end{aligned}
$$

where we used Fay's identity (45) in the second equality. On use of (52) in (51), it is found that

$$
\alpha\left(\theta_{1}, \theta\right) \frac{\partial X_{\theta_{1}, \theta_{3}, \theta}}{\partial \theta}+\beta\left(\theta_{3}, \theta\right) \frac{\partial Y_{\theta_{1}, \theta_{3}, \theta}}{\partial \theta}=0 .
$$

Suppose now that we fix the two parameters $\theta_{1}$ and $\theta_{3}$. Then $\left(X_{\theta_{1}, \theta_{3}, \theta}, Y_{\theta_{1}, \theta_{3}, \theta}\right)$ depends only on the single parameter $\theta$ and the tangent at $\left(X_{\theta_{1}, \theta_{3}, \theta}, Y_{\theta_{1}, \theta_{3}, \theta}\right)$ when viewed as a function of $\theta$ is defined as the set of $(X, Y)$ satisfying

$$
Y-Y_{\theta_{1}, \theta_{3}, \theta}=\frac{\partial Y_{\theta_{1}, \theta_{3}, \theta} / \partial \theta}{\partial X_{\theta_{1}, \theta_{3}, \theta} / \partial \theta}\left(X-X_{\theta_{1}, \theta_{3}, \theta}\right) .
$$

If we now make use of both (53) and (49) the tangent line (54) is equivalent to

$$
\alpha\left(\theta_{1}, \theta\right) X+\beta\left(\theta_{3}, \theta\right) Y=1
$$

It is helpful to visualize this: Fig. 5 shows examples of these tangent lines. The red line shows the collection of points $\left(X_{\theta_{1}, \theta_{3}, \theta}, Y_{\theta_{1}, \theta_{3}, \theta}\right)$ where both $\theta_{1}$ and $\theta_{3}$ are fixed but where $\theta$ is varying; the blue lines in Fig. 5, given by (55), are tangent to those red lines. Because of the angular conditions (44), $\theta$ varies between $\left|\theta_{1}\right|$ and $\pi-\left|\pi-\theta_{3}\right|$.

The important point, however, is that because the symmetry case shown in Fig. 2 corresponds to $\theta_{1}=0$ and $\theta_{3}=\pi$, the two inequalities in (5) are equivalent to the two statements

$$
\begin{aligned}
& X+Y \leq 1, \\
& \alpha_{\theta} X+\beta_{\theta} Y \geq 1, \quad \alpha_{\theta} \equiv \alpha(0, \theta), \quad \beta_{\theta} \equiv \beta(\pi, \theta),
\end{aligned}
$$

for all pairs $(X, Y)$ and $0<\theta<\pi$. The first statement is that all pairs $(X, Y)$ for $\rho \geq 0$ lie on or below the line $X+Y=1$ relevant to the classical van der Pauw case $\rho=0$; the second statement is that all pairs $(X, Y)$ for $\rho \geq 0$ lie above a $\theta$-parametrized curve 

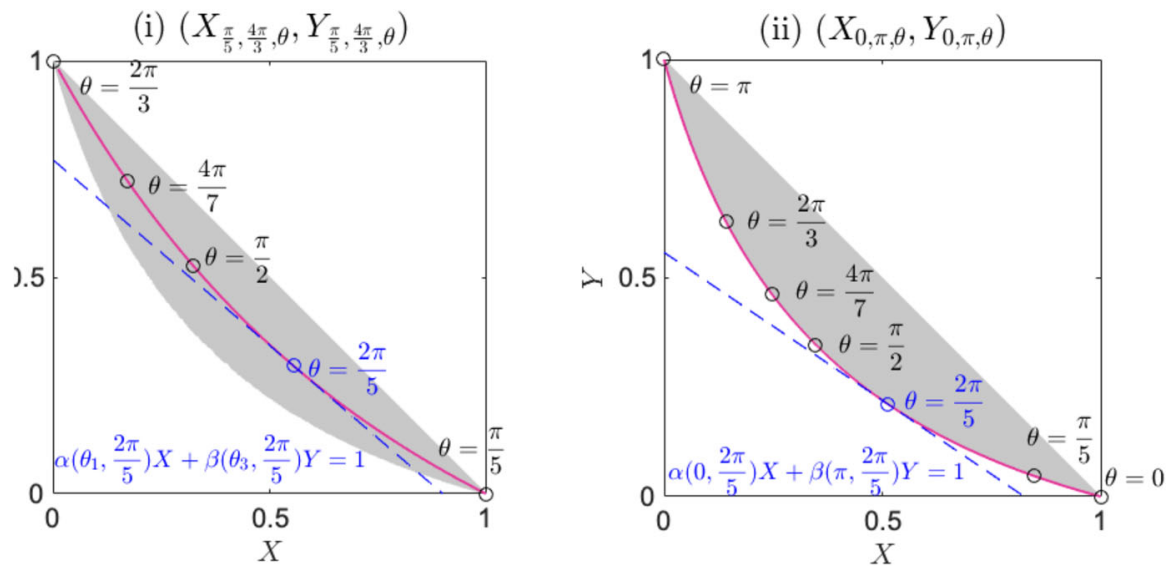

Fig. 5 The red lines are collections of data points $\left(X_{\theta_{1}, \theta_{3}, \theta}, Y_{\theta_{1}, \theta_{3}, \theta}\right)$ with both $\theta_{1}$ and $\theta_{3}$ fixed and only parameter $\theta$ changed. The blue lines, given by (55), are tangent to the red lines at $\left(X_{\theta_{1}, \theta_{3}, \theta}, Y_{\theta_{1}, \theta_{3}, \theta}\right)$. When $\theta_{1}=0$ and $\theta_{3}=\pi$, the red line is a tangent to a lower envelope

defined for $\theta_{1}=0$ and $\theta_{3}=\pi$ where the parameter $\theta$ corresponds geometrically to points arranged with the symmetry shown in Fig. 2.

The strategy is to prove (56) and (57) for all $(X, Y)$ by considering the maximum and minimum values of the coefficient functions $\alpha\left(\theta_{1}, \theta\right)$ and $\beta\left(\theta_{3}, \theta\right)$ subject to the condition (44). From the integrated Fay identity (49),

$$
\begin{gathered}
\min _{-\theta<\theta_{1}<\theta}\left(\alpha\left(\theta_{1}, \theta\right)\right) X_{\theta_{1}, \theta_{3}, \theta}+\min _{\theta<\theta_{3}<2 \pi-\theta}\left(\beta\left(\theta_{3}, \theta\right)\right) Y_{\theta_{1}, \theta_{3}, \theta} \\
\leq \alpha\left(\theta_{1}, \theta\right) X_{\theta_{1}, \theta_{3}, \theta}+\beta\left(\theta_{3}, \theta\right) Y_{\theta_{1}, \theta_{3}, \theta}=1, \\
\max _{-\theta<\theta_{1}<\theta}\left(\alpha\left(\theta_{1}, \theta\right)\right) X_{\theta_{1}, \theta_{3}, \theta}+\max _{\theta<\theta_{3}<2 \pi-\theta}\left(\beta\left(\theta_{3}, \theta\right)\right) Y_{\theta_{1}, \theta_{3}, \theta} \\
\geq \alpha\left(\theta_{1}, \theta\right) X_{\theta_{1}, \theta_{3}, \theta}+\beta\left(\theta_{3}, \theta\right) Y_{\theta_{1}, \theta_{3}, \theta}=1 .
\end{gathered}
$$

The idea is to show that (58) is equivalent to (56), and (59) is equivalent to (57).

To study the extrema of $\alpha\left(\theta_{1}, \theta\right)$ and $\beta\left(\theta_{1}, \theta\right)$, we define the function

$$
g_{\theta}(\eta) \equiv \frac{1}{2 \pi} \int_{0}^{2 \pi} \frac{P\left(\rho e^{\mathrm{i}(\phi-\eta)}\right) P\left(\rho e^{\mathrm{i}(\phi+\eta)}\right)}{P\left(\rho e^{\mathrm{i}(\phi-\theta)}\right) P\left(\rho e^{\mathrm{i}(\phi+\theta)}\right)} d \phi=\frac{1}{2 \pi} \int_{0}^{2 \pi} \mathcal{G}_{\theta, \eta}\left(\rho e^{\mathrm{i} \phi}\right) d \phi
$$

where $0 \leq \eta \leq 2 \pi$ and the integrand is defined as

$$
\mathcal{G}_{\theta, \eta}(\zeta) \equiv \frac{P(\zeta / \mu) P(\zeta \mu)}{P(\zeta / \nu) P(\zeta \nu)}, \quad \mu \equiv e^{\mathrm{i} \eta}, \quad \nu \equiv e^{\mathrm{i} \theta}
$$


(i) $\theta_{1}=\theta-2 \epsilon, \theta_{3}=2 \pi-\theta-\epsilon, \theta=\frac{\pi}{3}, \epsilon=10^{-3}$
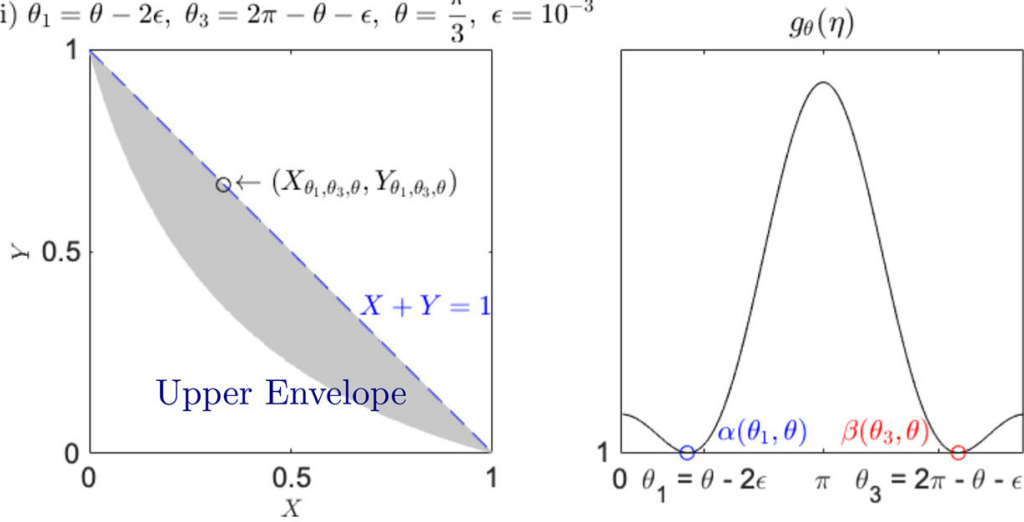

(ii) $\theta_{1}=\frac{\pi}{9}, \theta_{3}=\frac{5 \pi}{4}, \theta=\frac{\pi}{3}$
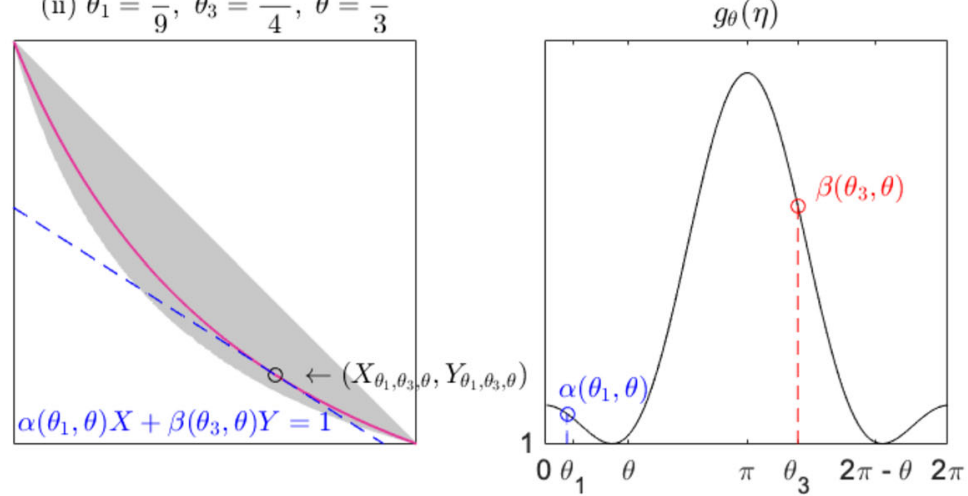

(iii) $\theta_{1}=0, \theta_{3}=\pi, \theta=\frac{\pi}{3}$
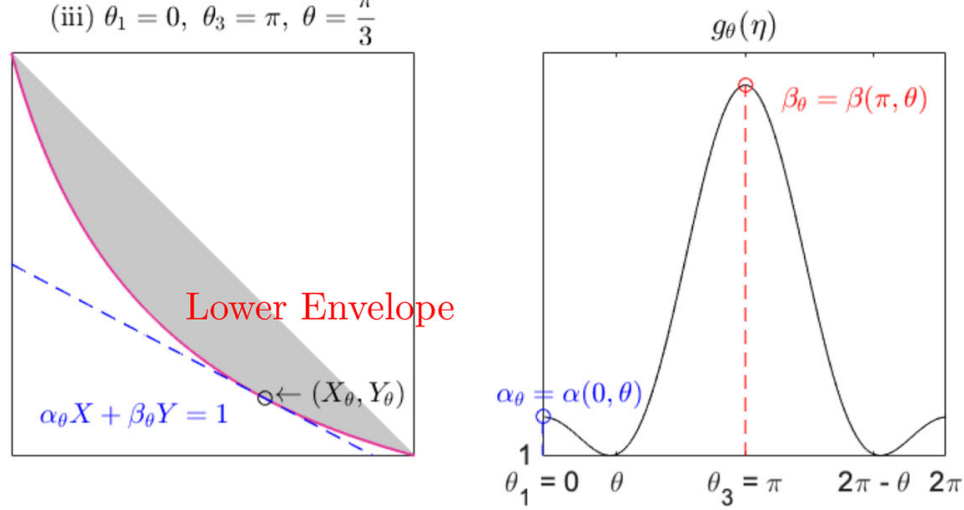

Fig. 6 Visualizing the relationship between tangent lines and the function $g_{\theta}(\eta)$ defined in (60). Red lines show the curves produced by changing only $\theta$, and blue lines are tangents to the envelope at $\left(X_{\theta_{1}, \theta_{3}, \theta}, Y_{\theta_{1}, \theta_{3}, \theta}\right)$. When $\alpha\left(\theta_{1}, \theta\right)$ and $\beta\left(\theta_{3}, \theta\right)$ are minimised, the tangent line corresponds to $X+Y=1$. When $\alpha\left(\theta_{1}, \theta\right)$ and $\beta\left(\theta_{3}, \theta\right)$ are maximised, the line becomes a tangent line to the lower envelope at $\left(X_{\theta}, Y_{\theta}\right)$, where $X_{\theta} \equiv X_{0, \pi, \theta}$ and $Y_{\theta} \equiv Y_{0, \pi, \theta}$ 
From the definitions (50) it is clear that the two coefficients functions in (49) can be written in terms of this single function:

$$
\alpha\left(\theta_{1}, \theta\right)=g_{\theta}\left(\theta_{1}\right), \quad \beta\left(\theta_{3}, \theta\right)=g_{\theta}\left(\theta_{3}\right)
$$

Analysis of this function $g_{\theta}(\eta)$ provides the key to the proofs of the conjectures.

Before presenting the details, the strategy explained in (58)-(59) is illustrated in Fig. 6. By minimising both $\alpha\left(\theta_{1}, \theta\right)$ and $\beta\left(\theta_{3}, \theta\right)$, the upper envelope $X+Y=1$ is obtained. In contrast, by maximising both $\alpha\left(\theta_{1}, \theta\right)$ and $\beta\left(\theta_{3}, \theta\right)$, a tangent line to the lower envelope is obtained.

It is important to mention that because of the definition of $P(\zeta)$,

$$
P\left(\rho e^{\mathrm{i} \phi}\right)=\prod_{n=1}^{\infty}\left(1+\rho^{4 n-2}-2 \rho^{2 n-1} \cos \phi\right)>0,
$$

which means $\mathcal{G}_{\theta, \eta}\left(\rho e^{\mathrm{i} \phi}\right)$ is real and positive. By a log-sum and sum-log inequality, we obtain

$$
\begin{aligned}
\log g_{\theta}(\eta)= & \log \left[\frac{1}{2 \pi} \int_{0}^{2 \pi} \frac{P\left(\rho e^{\mathrm{i}(\phi-\eta)}\right) P\left(\rho e^{\mathrm{i}(\phi+\eta)}\right)}{P\left(\rho e^{\mathrm{i}(\phi-\theta)}\right) P\left(\rho e^{\mathrm{i}(\phi+\theta)}\right)} d \phi\right] \\
\geq & \frac{1}{2 \pi} \int_{0}^{2 \pi} \log \frac{P\left(\rho e^{\mathrm{i}(\phi-\eta)}\right) P\left(\rho e^{\mathrm{i}(\phi+\eta)}\right)}{P\left(\rho e^{\mathrm{i}(\phi-\theta)}\right) P\left(\rho e^{\mathrm{i}(\phi+\theta)}\right)} d \phi \\
= & \frac{1}{2 \pi} \int_{0}^{2 \pi}\left[\log \left(P\left(\rho e^{\mathrm{i}(\phi-\eta)}\right)\right)+\log \left(P\left(\rho e^{\mathrm{i}(\phi+\eta)}\right)\right)\right. \\
& -\log \left(P\left(\rho e^{\mathrm{i}(\phi-\theta)}\right)-\log \left(P\left(\rho e^{\mathrm{i}(\phi+\theta)}\right)\right)\right] d \phi=0 .
\end{aligned}
$$

Thus, we can conclude that $g_{\theta}(\eta) \geq 1$ for all $\theta$ and $\eta$. We will use this in the next section to analyse the behavior of the function $g_{\theta}(\eta)$.

\section{Analysis of the Functions $g_{\theta(\eta)}$ and $\frac{\partial g_{\theta}}{\partial \eta}$}

This section studies the behavior of the function $g_{\theta}(\eta)$. First, we propose an alternative expression of $g_{\theta}(\eta)$ to be:

$$
g_{\theta}(\eta)=\frac{P(\nu / \mu) P(\mu \nu)}{\hat{P}(1) P\left(\nu^{2}\right)}[K(\mu / \nu)-K(\mu \nu)],
$$


where we recall that $\mu=e^{\mathrm{i} \eta}$ and $\nu=e^{\mathrm{i} \theta}$. This expression is useful for the analysis of $g_{\theta}(\eta)$.

To explain the derivation of (65) we note that the integrand $\mathcal{G}_{\theta, \eta}(\zeta)$ defined in (61) has two simple poles at $\zeta=v$ and $\zeta=1 / v$ and is readily confirmed, on use of (19), to be loxodromic. The function $\mathcal{G}_{\theta, \eta}(\zeta)$ can therefore also be written as

$$
\mathcal{G}_{\theta, \eta}(\zeta)=\frac{P(\zeta / \mu) P(\zeta \mu)}{P(\zeta / \nu) P(\zeta \nu)}=c_{1}[K(\zeta / \nu)-K(\zeta \nu)]+c_{2}
$$

where $c_{1}, c_{2} \in \mathbb{C}$. Since $K(\zeta)$ is a logarithmic derivative of $P(\zeta)$ and has functional properties (21), the right hand side of (66) is also a loxodromic function and has two simple poles at $\zeta=v$ and $\zeta=1 / \nu$. The coefficients $c_{1}$ and $c_{2}$ are determined by considering the limits $\zeta \rightarrow v$ and $\zeta \rightarrow \mu$,

$$
c_{2}=-c_{1}[K(\mu / \nu)-K(\mu \nu)], \quad c_{2}=\frac{P(\nu / \mu) P(\mu \nu)}{\hat{P}(1) P\left(v^{2}\right)}[K(\mu / \nu)-K(\mu \nu)] .
$$

A Liouville-type argument then confirms the equivalence of the two expressions for $\mathcal{G}_{\theta, \eta}(\zeta)$ in (66). We therefore conclude by using (60) and (66) that

$$
\begin{aligned}
g_{\theta}(\eta) & =\frac{1}{2 \pi} \int_{0}^{2 \pi} \mathcal{G}_{\theta, \eta}\left(\rho e^{\mathrm{i} \phi}\right) d \phi \\
& =\frac{1}{2 \pi} \int_{0}^{2 \pi}\left(c_{1}\left[K\left(\rho e^{\mathrm{i}(\phi-\theta)}\right)-K\left(\rho e^{\mathrm{i}(\phi+\theta)}\right)\right]+c_{2}\right) d \phi=c_{2},
\end{aligned}
$$

which is precisely (65).

Now, we consider the behavior of the function $g_{\theta}(\eta)$ by exploiting the derivative of $g_{\theta}(\eta)$ :

$$
\frac{\partial g_{\theta}}{\partial \eta}=\frac{\partial \mu}{\partial \eta} \frac{\partial g_{\theta}}{\partial \mu}=\mathrm{i} \frac{P(\nu / \mu) P(\mu \nu)}{\hat{P}(1) P\left(\nu^{2}\right)}(L(\mu / \nu)-L(\mu \nu))+\mathrm{i} g_{\theta}[K(\mu \nu)-K(\nu / \mu)]
$$

The function $L(\mu / \nu)-L(\mu \nu)$ is a loxodromic function as a function of $\mu$, and has two second-order poles at $\mu=v$ and $\mu=1 / v$. Similar to the expression of $g_{\theta}(\eta)$, we propose another expression of $L(\mu / \nu)-L(\mu \nu)$ given by

$$
L(\mu / \nu)-L(\mu \nu)=\frac{\hat{P}(1)^{2} P\left(\mu^{2}\right) P\left(v^{2}\right)}{P(\nu / \mu) P(\mu / \nu) P(\mu \nu)^{2}} .
$$


To establish this expression, consider a new function

$$
\mathcal{L}(\mu) \equiv L(\mu / \nu)-L(\mu \nu)-\frac{\hat{P}(1)^{2} P\left(\mu^{2}\right) P\left(v^{2}\right)}{P(\nu / \mu) P(\mu / \nu) P(\mu \nu)^{2}}
$$

The function $\mathcal{L}(\mu)$ is shown to be a loxodromic function by using the functional properties (19) and (21) as follows:

$$
\begin{aligned}
\mathcal{L}\left(\rho^{2} \mu\right) & =L\left(\rho^{2} \mu / \nu\right)-L\left(\rho^{2} \mu \nu\right)-\frac{\hat{P}(1)^{2} P\left(\rho^{4} \mu^{2}\right) P\left(v^{2}\right)}{P\left(\nu / \rho^{2} \mu\right) P\left(\rho^{2} \mu / \nu\right) P\left(\rho^{2} \mu \nu\right)^{2}} \\
& =L(\mu / \nu)-L(\mu \nu)-\frac{\hat{P}(1)^{2}\left(\rho^{2} \mu^{4}\right)^{-1} P\left(\mu^{2}\right) P\left(v^{2}\right)}{\left(\rho^{2} \mu / \nu\right)^{-1} P(\nu / \mu)(\mu / \nu)^{-1} P(\mu / \nu)(\mu \nu)^{-2} P(\mu \nu)^{2}} \\
& =\mathcal{L}(\mu) .
\end{aligned}
$$

Furthermore, the asymptotic expansions of each term at $\mu=v$ and $\mu=1 / \nu$ are

$$
\begin{aligned}
L(\mu / \nu)-L(\mu \nu) & \sim-\frac{1}{(1-\mu / \nu)^{2}}+\frac{1}{1-\mu / v}+\mathcal{O}(1) \quad \text { at } \mu=v, \\
L(\mu / \nu)-L(\mu \nu) & \sim \frac{1}{(1-\mu \nu)^{2}}-\frac{1}{1-\mu \nu}+\mathcal{O}(1) \quad \text { at } \mu=1 / \nu, \\
\frac{\hat{P}(1)^{2} P\left(\mu^{2}\right) P\left(v^{2}\right)}{P(\nu / \mu) P(\mu / \nu) P(\mu \nu)^{2}} & \sim \frac{1}{(1-v / \mu)(1-\mu / v)}+\mathcal{O}(1) \\
& =-\frac{1}{(1-\mu / v)^{2}}+\frac{1}{1-\mu / v}+\mathcal{O}(1) \quad \text { at } \mu=v, \\
\frac{\hat{P}(1)^{2} P\left(\mu^{2}\right) P\left(v^{2}\right)}{P(\nu / \mu) P(\mu / \nu) P(\mu \nu)^{2}} & \sim \frac{1}{(1-\mu \nu)^{2}}-\frac{1}{1-\mu \nu}+\mathcal{O}(1) \quad \text { at } \mu=1 / \nu .
\end{aligned}
$$

Thus the two poles at $\nu$ and $1 / \nu$ are removable. We conclude that $\mathcal{L}(\mu)$ is a constant function, i.e., it is independent of $\mu$. In addition, we define another function

$$
\tilde{\mathcal{L}}(\nu) \equiv L(\mu / \nu)-L(\mu \nu)-\frac{\hat{P}(1)^{2} P\left(\mu^{2}\right) P\left(v^{2}\right)}{P(\nu / \mu) P(\mu / \nu) P(\mu \nu)^{2}}
$$

By similar arguments, the function $\tilde{\mathcal{L}}(v)$ can be seen to be a loxodromic function of $\nu$ with removable poles $v=\mu$ and $v=1 / \mu$. Thus, $\mathcal{L}(\mu)$ is independent of both $\mu$ and $\nu$.

On use of the functional property (21) of $L(\zeta)$, it can be verified that $\mathcal{L}(\rho)=0$, which means $\mathcal{L}(\mu)$ vanishes everywhere leading to expression (70). 

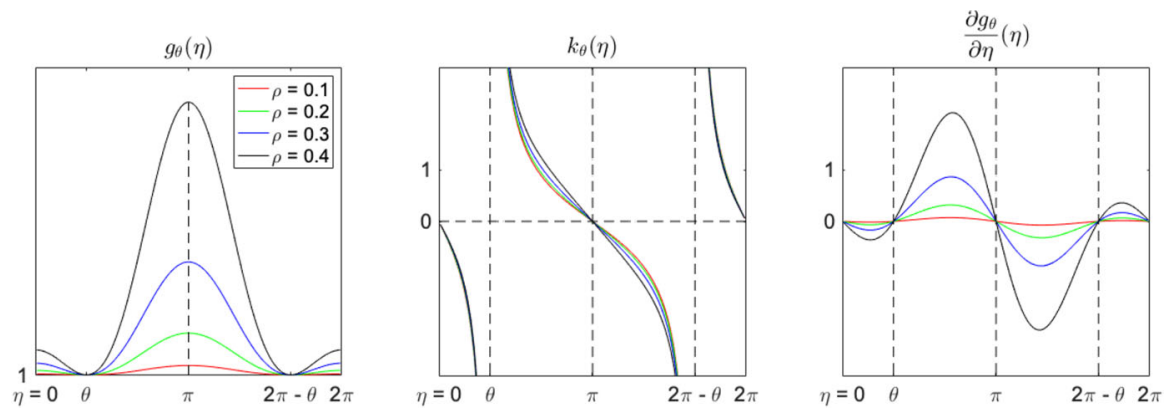

Fig. 7 Typical behavior of the function $g_{\theta}(\eta), k_{\theta}(\eta)$, and $\partial g_{\theta} / \partial \eta$. A local maximum of $g_{\theta}(\eta)$ is obtained when $\eta=0$ or $\eta=\pi$. A local minimum of $g(\eta)$ is obtained when $\eta=\theta$ or $2 \pi-\theta$

Expression (70) can now be used to analyse the behaviour of $g_{\theta}(\eta)$. By substituting (70) into (69),

$$
\begin{aligned}
\frac{\partial g_{\theta}}{\partial \eta} & =\mathrm{i}\left[\frac{\hat{P}(1) P\left(\mu^{2}\right)}{P(\mu / \nu) P(\mu \nu)}+g_{\theta}(\eta)(K(\mu \nu)-K(\nu / \mu))\right] \\
& =\mathrm{i}(K(\mu \nu)-K(\nu / \mu))\left[g_{\theta}(\eta)-\frac{1}{K(\nu / \mu)-K(\mu \nu)} \frac{\hat{P}(1) P\left(\mu^{2}\right)}{P(\mu / \nu) P(\mu \nu)}\right] \\
& =\mathrm{i}(K(\mu \nu)-K(\nu / \mu))\left[g_{\theta}(\eta)-\frac{1}{g_{\eta}(\theta)}\right] .
\end{aligned}
$$

Since, from (64), $g_{\theta}(\eta) \geq 1$ and $g_{\eta}(\theta) \geq 1$ then

$$
g_{\theta}(\eta)-\frac{1}{g_{\eta}(\theta)} \geq 0
$$

The sign of the derivative (75) is therefore determined by the function

$$
k_{\theta}(\eta) \equiv \mathrm{i}(K(\mu \nu)-K(\nu / \mu)) .
$$

The derivative of $k_{\theta}(\eta)$ with respect to $\eta$ is

$$
\frac{\partial k_{\theta}}{\partial \eta}=-(L(\mu \nu)+L(\nu / \mu))
$$

But it can also be shown that $L\left(e^{\mathrm{i} \phi}\right)$ for $0<\phi<2 \pi$ is real and positive- the proof is given in Appendix A. This means that

$$
\frac{\partial k_{\theta}}{\partial \eta}<0, \quad 0<\eta<2 \pi
$$


It is straightforward to check that $k_{\theta}(0)=k_{\theta}(\pi)=0$ and consequently, using continuity arguments,

$$
\begin{aligned}
\lim _{\eta \rightarrow \theta-0} k_{\theta}(\eta) & =-\infty, & \lim _{\eta \rightarrow \theta+0} k_{\theta}(\eta) & =+\infty, \\
\lim _{\eta \rightarrow 2 \pi-\theta-0} k_{\theta}(\eta) & =-\infty, & \lim _{\eta \rightarrow 2 \pi-\theta+0} k_{\theta}(\eta) & =+\infty .
\end{aligned}
$$

With this information we can determine the maxima and the minima of $g_{\theta}(\eta)$. The local minima occur when $\eta=\theta$ or $\eta=2 \pi-\theta$. By the definition (60),

$$
g_{\theta}(\theta)=g_{\theta}(2 \pi-\theta)=1
$$

On the contrary, local maxima occur when $\eta=0$ and $\eta=\pi$. Recall that this case corresponds to the symmetric choice of points with parameter $\theta$. Figure 7 shows the typical behaviour of the function $g_{\theta}(\eta)$ for a range of $\rho$ values; recall that $P(\zeta)$, and hence $g_{\theta}(\eta)$ and $k_{\theta}(\eta)$ depend on the parameter $\rho$ although this dependence is hidden in our notation.

\section{Proof of the Two Conjectures (56)-(57)}

We will now prove the two conjectures (56) and (57) using what we have established about the behavior of $g_{\theta}(\eta)$. From (58) and (82), we can conclude that

$$
X_{\theta_{1}, \theta_{3}, \theta}+Y_{\theta_{1}, \theta_{3}, \theta} \leq 1
$$

for all $\left(X_{\theta_{1}, \theta_{3}, \theta}, Y_{\theta_{1}, \theta_{3}, \theta}\right)$. This is equivalent to the conjecture (56).

The maxima of $\alpha\left(\theta_{1}, \theta\right)$ and $\beta\left(\theta_{3}, \theta\right)$ occur when $\theta_{1}=0$ and $\theta_{3}=\pi$. Hence, from (59), we conclude that

$$
\alpha_{\theta} X_{\theta_{1}, \theta_{3}, \theta}+\beta_{\theta} Y_{\theta_{1}, \theta_{3}, \theta} \geq 1
$$

The inequality (84) is not, however, equivalent to the inequality (57). What has been proven in (84) is that for $0<\theta<\pi$, the pair $\left(X_{\theta_{1}, \theta_{3}, \theta}, Y_{\theta_{1}, \theta_{3}, \theta}\right)$ is above the tangent at $\left(X_{0, \pi, \theta}, Y_{0, \pi, \theta}\right)$ on the lower envelope. Thus, for the final step, we need to prove that for another $\theta^{\prime} \neq \theta$, the pair $\left(X_{\theta_{1}, \theta_{3}, \theta}, Y_{\theta_{1}, \theta_{3}, \theta}\right)$ lies above the tangent at $\left(X_{0, \pi, \theta^{\prime}}, Y_{0, \pi, \theta^{\prime}}\right)$. The condition is equivalent to

$$
\alpha_{\theta^{\prime}} X_{\theta_{1}, \theta_{3}, \theta}+\beta_{\theta^{\prime}} Y_{\theta_{1}, \theta_{3}, \theta} \geq 1
$$

Notice that because of the choice of angular coordinates (44) the range of $\theta_{1}$ and $\theta_{3}$ are related to $\theta$, but $\theta^{\prime}$ should be chosen independently of $\theta_{1}$ and $\theta_{3}$ since we need it to parametrize the whole of the lower envelope.

The procedure to prove (85) can be divided into two steps.

In the first step (Step 1) we find a specific $\tilde{\theta}$ such that $\alpha_{\theta^{\prime}} \geq \alpha\left(\theta_{1}, \tilde{\theta}\right)$ and $\beta_{\theta^{\prime}} \geq$ $\beta\left(\theta_{3}, \tilde{\theta}\right)$. This step can be viewed as a movement from a point on the lower envelope 
(the set of 3 blue curves in Fig. 8) to a point on another curve (the red curve in Fig. 8) on which the point $\left(X_{\theta_{1}, \theta_{3}, \theta}, Y_{\theta_{1}, \theta_{3}, \theta}\right)$ lies.

In the next step (Step 2) we make a use of a log-sum and sum-log inequality to prove that

$$
\alpha\left(\theta_{1}, \tilde{\theta}\right) X_{\theta_{1}, \theta_{3}, \theta}+\beta\left(\theta_{3}, \tilde{\theta}\right) Y_{\theta_{1}, \theta_{3}, \theta} \geq 1
$$

First let us explain Step 1. For an arbitrary value of $\theta^{\prime}$ with $0<\theta^{\prime}<\pi$, there are three possible cases for the set $\left(\theta^{\prime}, \theta_{1}, \theta_{3}\right)$. The three cases are illustrated in Fig. 8: they can be understood as corresponding to the lower envelope being split into three parts: the three blue curve segments sitting on the lower envelope in Fig. 8 as shown in top, middle, and bottom of this figure. These will be called cases 1, 2a and 2b, respectively. The red solid curve on each Fig. 8 is drawn by changing $\theta$ from $\left|\theta_{1}\right|$ to $\pi-\left|\pi-\theta_{3}\right|$ while $\theta_{1}$ and $\theta_{3}$ are fixed. The range of $\theta$ comes from the condition of angular coordinates (44). The reason for the separation into three cases is that when $\theta^{\prime}<\left|\theta_{1}\right|$ or $\pi-\left|\pi-\theta_{3}\right|<\theta^{\prime}$, an auxiliary point $\left(X_{\theta_{1}, \theta_{3}, \theta^{\prime}}, Y_{\theta_{1}, \theta_{3}, \theta^{\prime}}\right)$ does not lie on the red curve. Based on the argument above, we therefore assign a value $\tilde{\theta}$ to a value of $\theta^{\prime}$ according to the following three conditions:

- Case 1: When $\left|\theta_{1}\right| \leq \theta^{\prime} \leq \pi-\left|\pi-\theta_{3}\right|$, which means that the point $\left(X_{\theta^{\prime}}, Y_{\theta^{\prime}}\right)$ marked as "+" in the top right of Fig. 8 lies on the blue solid curve, an auxiliary point $\left(X_{\theta_{1}, \theta_{3}, \theta^{\prime}}, Y_{\theta_{1}, \theta_{3}, \theta^{\prime}}\right)$ also lies on the red curve marked as " $x$ ". From the behavior of $g_{\theta^{\prime}}(\eta)$, we can see that $\alpha_{\theta^{\prime}} \geq \alpha\left(\theta_{1}, \theta^{\prime}\right)$ and $\beta_{\theta^{\prime}} \geq \beta\left(\theta_{3}, \theta^{\prime}\right)$. In this case, we choose $\tilde{\theta}=\theta^{\prime}$.

- Case 2a: When $\theta^{\prime}<\left|\theta_{1}\right|$ as shown in the middle of Fig. 8, an auxiliary point $\left(X_{\theta_{1}, \theta_{3}, \theta^{\prime}}, Y_{\theta_{1}, \theta_{3}, \theta^{\prime}}\right)$ does not lie on the red curve. In this case, the end point of the red curve is chosen, that is, we make the choice $\tilde{\theta}=\left|\theta_{1}\right|$. From the behavior of $g_{\theta}(\eta)$, it is apparent that $\alpha_{\theta^{\prime}} \geq \alpha\left(\theta_{1},\left|\theta_{1}\right|\right)=1$. Because of the angular condition (44) such that $\left|\theta_{1}\right|<\theta_{3}<2 \pi-\left|\theta_{1}\right|$ and because $\beta_{\theta}$ is a monotonically decreasing function with respect to $\theta$ for $0<\theta<\pi$ (Appendix $\mathrm{B}$ ), $\beta_{\theta^{\prime}}>\beta_{\left|\theta_{1}\right|}=\beta\left(\pi,\left|\theta_{1}\right|\right) \geq$ $\beta\left(\theta_{3},\left|\theta_{1}\right|\right)$.

- Case 2b: This is the same as case $2 \mathrm{a}$ but refers to the other end of the red curve. When $\pi-\left|\pi-\theta_{3}\right|<\theta^{\prime}$ as shown in the bottom of Fig. 8, a point $\left(X_{\theta_{1}, \theta_{3}, \theta^{\prime}}, Y_{\theta_{1}, \theta_{3}, \theta^{\prime}}\right)$ does not lie on the red curve (cf: Case $2 \mathrm{a}$ ). In this case, the opposite boundary point of the red curve is now chosen, which means that we choose $\tilde{\theta}=\pi-\left|\pi-\theta_{3}\right|$. From the behavior of $g_{\theta}(\eta)$, it is apparent that $\beta_{\theta^{\prime}} \geq \beta\left(\theta_{3}, \pi-\left|\pi-\theta_{3}\right|\right)=1$. Because $\pi-\left|\pi-\theta_{3}\right|<\theta^{\prime},\left|\theta_{1}\right|<\pi-\left|\pi-\theta_{3}\right|$, and because $\alpha_{\theta}$ is a monotonically increasing function with respect to $\theta$ for $0<\theta<\pi$ (Appendix B), $\alpha_{\theta^{\prime}}>\alpha_{\pi-\left|\pi-\theta_{3}\right|} \geq$ $\alpha\left(\theta_{1}, \pi-\left|\pi-\theta_{3}\right|\right)$.

In all cases, it is possible to find a specific $\tilde{\theta}$ such that $\alpha_{\theta^{\prime}} \geq \alpha\left(\theta_{1}, \tilde{\theta}\right)$ and $\beta_{\theta^{\prime}} \geq$ $\beta\left(\theta_{3}, \tilde{\theta}\right)$. Thus, we obtain the important result that

$$
\alpha_{\theta^{\prime}} X_{\theta_{1}, \theta_{3}, \theta}+\beta_{\theta^{\prime}} Y_{\theta_{1}, \theta_{3}, \theta} \geq \alpha\left(\theta_{1}, \tilde{\theta}\right) X_{\theta_{1}, \theta_{3}, \theta}+\beta\left(\theta_{3}, \tilde{\theta}\right) Y_{\theta_{1}, \theta_{3}, \theta}
$$

This facilitates Step 2 where we use a log-sum and sum-log inequality for the right hand side of (87) as follows: 
(i) Case 1

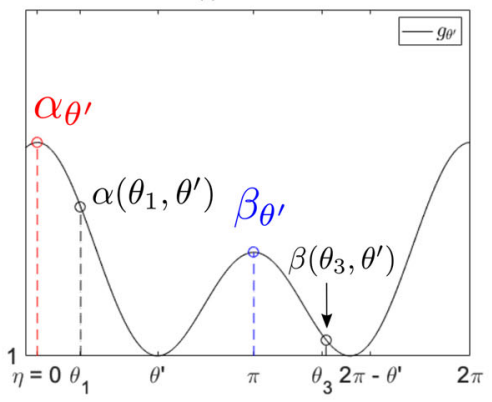

(ii) Case 2a

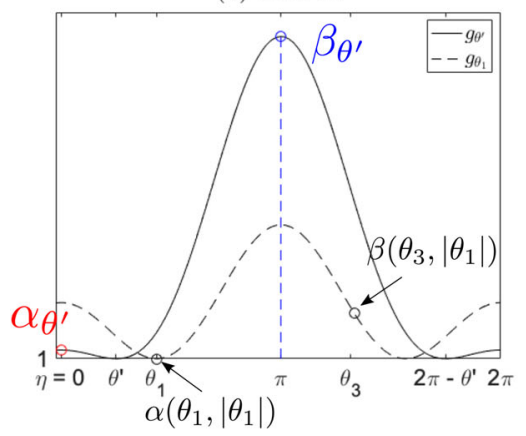

(iii) Case 2b

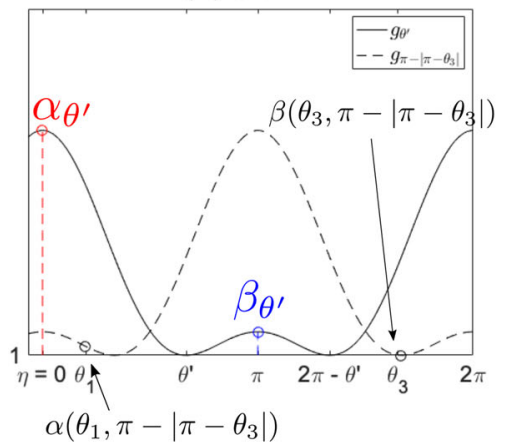

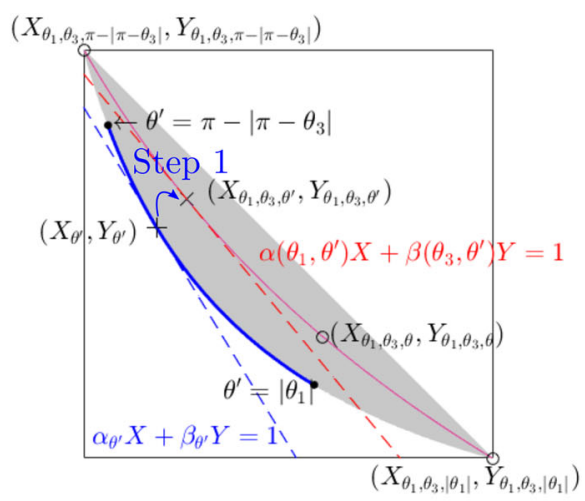

$\left(X_{\theta_{1}, \theta_{3}, \pi-\left|\pi-\theta_{3}\right|}, Y_{\theta_{1}, \theta_{3}, \pi-\left|\pi-\theta_{3}\right|}\right)$
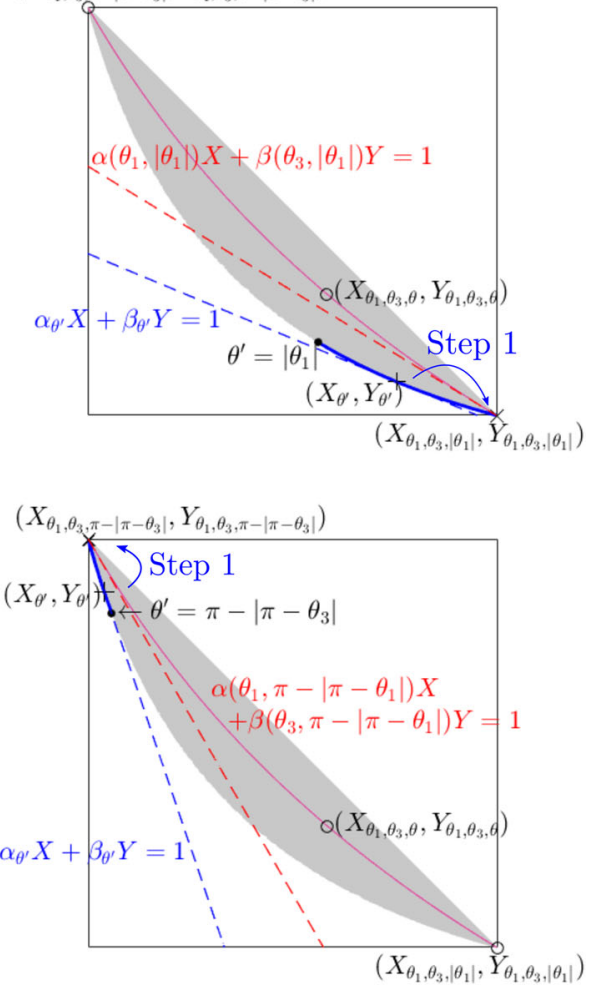

Fig. 8 Three possible cases for the set $\left(\theta^{\prime}, \theta_{1}, \theta_{3}\right)$. (i) Case 1: $\alpha_{\theta^{\prime}} \geq \alpha\left(\theta_{1}, \theta^{\prime}\right)$ and $\beta_{\theta^{\prime}} \geq \beta\left(\theta_{3}, \theta^{\prime}\right)$. (ii) Case 2a: Because $\left|\theta_{1}\right|>\theta^{\prime}$, we choose $\tilde{\theta}=\left|\theta_{1}\right|$. We can see that $\alpha_{\theta^{\prime}} \geq \alpha\left(\theta_{1},\left|\theta_{1}\right|\right)=1$ and $\beta_{\theta^{\prime}}>\beta\left(\theta_{3},\left|\theta_{1}\right|\right)$. (iii) Case $2 \mathrm{~b}$ : Because $\left|\pi-\theta_{3}\right|>\pi-\theta^{\prime}$, we choose $\tilde{\theta}=\pi-\left|\pi-\theta_{3}\right|$. We can see that $\alpha_{\theta^{\prime}} \geq \alpha\left(\theta_{1}, \pi-\left|\pi-\theta_{3}\right|\right)$ and $\beta_{\theta^{\prime}}>\beta\left(\theta_{3}, \pi-\left|\pi-\theta_{3}\right|\right)=1$

$$
\begin{aligned}
\log & {\left[\alpha\left(\theta_{1}, \tilde{\theta}\right) X_{\theta_{1}, \theta_{3}, \theta}+\beta\left(\theta_{3}, \tilde{\theta}\right) Y_{\theta_{1}, \theta_{3}, \theta}\right] } \\
& =\log \left[\frac{1}{2 \pi} \int_{0}^{2 \pi}\left(A\left(\theta_{1}, \tilde{\theta}, \rho e^{\mathrm{i} \phi}\right) X_{\theta_{1}, \theta_{3}, \theta}+B\left(\theta_{3}, \tilde{\theta}, \rho e^{\mathrm{i} \phi}\right) Y_{\theta_{1}, \theta_{3}, \theta}\right) d \phi\right]
\end{aligned}
$$




$$
\geq \frac{1}{2 \pi} \int_{0}^{2 \pi} \log \left[A\left(\theta_{1}, \tilde{\theta}, \rho e^{\mathrm{i} \phi}\right) X_{\theta_{1}, \theta_{3}, \theta}+B\left(\theta_{3}, \tilde{\theta}, \rho e^{\mathrm{i} \phi}\right) Y_{\theta_{1}, \theta_{3}, \theta}\right] d \phi .
$$

However the right hand side can be written

$$
\begin{aligned}
& \frac{1}{2 \pi} \int_{0}^{2 \pi} \log \left[X_{\theta_{1}, \theta_{3}, \theta}+\frac{B\left(\theta_{3}, \tilde{\theta}, \rho e^{\mathrm{i} \phi}\right)}{A\left(\theta_{1}, \tilde{\theta}, \rho e^{\mathrm{i} \phi}\right)} Y_{\theta_{1}, \theta_{3}, \theta}\right] d \phi+\frac{1}{2 \pi} \int_{0}^{2 \pi} \log A\left(\theta_{1}, \tilde{\theta}, \rho e^{\mathrm{i} \phi}\right) d \phi \\
& \quad=\frac{1}{2 \pi} \int_{0}^{2 \pi} \log \left[X_{\theta_{1}, \theta_{3}, \theta}+\frac{B\left(\theta_{3}, \theta, \rho e^{\mathrm{i} \phi}\right)}{A\left(\theta_{1}, \theta, \rho e^{\mathrm{i} \phi}\right)} Y_{\theta_{1}, \theta_{3}, \theta}\right] d \phi+\frac{1}{2 \pi} \int_{0}^{2 \pi} \log A\left(\theta_{1}, \theta, \rho e^{\mathrm{i} \phi}\right) d \phi \\
& \quad=\frac{1}{2 \pi} \int_{0}^{2 \pi} \log \left[A\left(\theta_{1}, \theta, \rho e^{\mathrm{i} \phi}\right) X_{\theta_{1}, \theta_{3}, \theta}+B\left(\theta_{3}, \theta, \rho e^{\mathrm{i} \phi}\right) Y_{\theta_{1}, \theta_{3}, \theta}\right] d \phi=0,
\end{aligned}
$$

where we used the fact that the integral of $\log A\left(\theta_{1}, \theta, \rho e^{\mathrm{i} \phi}\right)$ is

$$
\begin{aligned}
& \frac{1}{2 \pi} \int_{0}^{2 \pi} \log A\left(\theta_{1}, \theta, \rho e^{\mathrm{i} \mathrm{E}}\right) d \phi=\frac{1}{2 \pi} \int_{0}^{2 \pi} \log \left(\frac{P\left(\rho e^{\mathrm{i}\left(\phi-\theta_{1}\right)}\right) P\left(\rho e^{\mathrm{i}\left(\phi+\theta_{1}\right)}\right)}{P\left(\rho e^{\mathrm{i}(\phi-\theta)}\right) P\left(\rho e^{\mathrm{i}(\phi+\theta)}\right)}\right) d \phi \\
&= \frac{1}{2 \pi} \int_{0}^{2 \pi}\left[\log P\left(\rho e^{\mathrm{i}\left(\phi-\theta_{1}\right)}\right)+\log P\left(\rho e^{\mathrm{i}\left(\phi+\theta_{1}\right)}\right)\right. \\
&\left.-\log P\left(\rho e^{\mathrm{i}(\phi-\theta)}\right)-\log P\left(\rho e^{\mathrm{i}(\phi+\theta)}\right)\right] d \phi=0
\end{aligned}
$$

and the fact that

$$
\frac{B\left(\theta_{1}, \tilde{\theta}, \rho e^{\mathrm{i} \phi}\right)}{A\left(\theta_{3}, \tilde{\theta}, \rho e^{\mathrm{i} \phi}\right)}=\frac{B\left(\theta_{1}, \theta, \rho e^{\mathrm{i} \phi}\right)}{A\left(\theta_{3}, \theta, \rho e^{\mathrm{i} \phi}\right)}=\frac{P\left(\rho e^{\mathrm{i}\left(\phi-\theta_{3}\right)}\right) P\left(\rho e^{\mathrm{i}\left(\phi+\theta_{3}\right)}\right)}{P\left(\rho e^{\mathrm{i}\left(\phi-\theta_{1}\right)}\right) P\left(\rho e^{\mathrm{i}\left(\phi+\theta_{1}\right)}\right)}
$$

is independent of $\theta$ and $\tilde{\theta}$. In the last line, we have used the Fay trisecant identity (45). Putting all this together it has been shown that

$$
\log \left[\alpha\left(\theta_{1}, \tilde{\theta}\right) X_{\theta_{1}, \theta_{3}, \theta}+\beta\left(\theta_{3}, \tilde{\theta}\right) Y_{\theta_{1}, \theta_{3}, \theta}\right] \geq 0 .
$$

From (92) it follows that

$$
\alpha_{\theta^{\prime}} X_{\theta_{1}, \theta_{3}, \theta}+\beta_{\theta^{\prime}} Y_{\theta_{1}, \theta_{3}, \theta} \geq 1
$$

which means for $0<\theta<\pi, \alpha_{\theta} X+\beta_{\theta} Y \geq 1$ for all $(X, Y)$. We have proved all the conjectured features of the envelope structure observed by previous authors. 
We now summarize steps 1 and 2 geometrically with the aid of Fig. 8. In step 1 we found a choice of an auxiliary tangent line (the red line) to the pink curve on which $\left(X_{\theta_{1}, \theta_{3}, \theta}, Y_{\theta_{1}, \theta_{3}, \theta}\right)$ sits (and which corresponds to a fixed $\theta_{1}$ and $\theta_{3}$ ) above the tangential line on the lower envelope (the blue line) for the three segments of the lower envelope into which it naturally divides for any fixed $\theta_{1}$ and $\theta_{3}$ (i.e., cases $1,2 \mathrm{a}$ and $2 \mathrm{~b}$ ). In step 2 the point $\left(X_{\theta_{1}, \theta_{3}, \theta}, Y_{\theta_{1}, \theta_{3}, \theta}\right)$ is then shown to be above that red tangent line by using the log-sum and sum-log inequalities. We thus prove that $\left(X_{\theta_{1}, \theta_{3}, \theta}, Y_{\theta_{1}, \theta_{3}, \theta}\right)$ is above any point on the lower envelope.

\section{Discussion}

By introducing the prime function on the Schottky double of a conformally equivalent concentric annulus to any given doubly connected sample with a single uncharged hole, and by using an integrated form of an associated Fay trisecant identity expressed in terms of that prime function, we have shown how to prove some conjectures made in the literature on an observed envelope structure associated with pairs of 4-point resistance measurements in a van der Pauw procedure. More practical implications of our results are discussed elsewhere [19].

The van der Pauw method for a sample with two or more holes is, of course, of interest and several studies have recently appeared $[9,11]$. The theory of the prime function on domains of general finite connectivity has been expounded in a recent monograph [18] and the relevance of the prime function to expressing the complex potential for source/sink flows in such domains is already known [23]. At the same time, the Fay trisecant identity also carries over to higher genus Riemann surfaces, where the Fay identity can be written down in terms of the so-called prime form on those surfaces and associated theta functions [24]. Therefore, while we have focused here on what is the simplest non-trivial case of a holey sample, we expect all the ideas to be extendible to the case of a sample with any finite number of holes. The details, however, remain to be worked out and constitute an interesting future challenge.

Open Access This article is licensed under a Creative Commons Attribution 4.0 International License, which permits use, sharing, adaptation, distribution and reproduction in any medium or format, as long as you give appropriate credit to the original author(s) and the source, provide a link to the Creative Commons licence, and indicate if changes were made. The images or other third party material in this article are included in the article's Creative Commons licence, unless indicated otherwise in a credit line to the material. If material is not included in the article's Creative Commons licence and your intended use is not permitted by statutory regulation or exceeds the permitted use, you will need to obtain permission directly from the copyright holder. To view a copy of this licence, visit http://creativecommons.org/licenses/by/4.0/. 


\section{Appendix}

\section{A The Positiveness of $L\left(e^{i \phi}\right)$ for $0<\phi<2 \pi$}

First, we consider the derivative of $L(\zeta)$ defined in (20) as follows:

$$
M(\zeta) \equiv \zeta \frac{\partial L}{\partial \zeta}
$$

Because we have another representation of $L(\mu / \nu)-L(\mu \nu)$ as stated in (70), we can consider a limit of each side:

$$
\lim _{\nu \rightarrow 1} \frac{L(\mu / v)-L(\mu \nu)}{1-v}=\mu \lim _{\nu \rightarrow 1}\left(\frac{1}{v} \frac{L(\mu / v)-L(\mu)}{\mu / v-\mu}+\frac{L(\mu)-L(\mu \nu)}{\mu-\mu \nu}\right)=2 M(\mu) .
$$

The right hand side is, from the definition in (18),

$$
\lim _{\nu \rightarrow 1} \frac{1}{1-v} \frac{\hat{P}(1)^{2} P\left(\mu^{2}\right) P\left(v^{2}\right)}{P(\nu / \mu) P(\mu / \nu) P(\mu \nu)^{2}}=2 \frac{\hat{P}(1)^{3} P\left(\mu^{2}\right)}{P(1 / \mu) P(\mu)^{3}} .
$$

We therefore find an alternative representation of $M(\zeta)$ :

$$
M(\zeta)=\frac{\hat{P}(1)^{3} P\left(\zeta^{2}\right)}{P(1 / \zeta) P(\zeta)^{3}}
$$

Consequently $M\left(e^{\mathrm{i} \phi}\right)$ is found to be

$$
M\left(e^{\mathrm{i} \phi}\right)=\frac{\hat{P}(1)^{3} P\left(e^{2 \mathrm{i} \phi}\right)}{P\left(1 / e^{\mathrm{i} \phi}\right) P\left(e^{\mathrm{i} \phi}\right)^{3}}=\frac{\mathrm{i}}{8} C(\phi) \frac{\sin \phi}{\sin ^{4} \frac{\phi}{2}},
$$

where

$$
C(\phi)=\hat{P}(1)^{3} \prod_{n=1}^{\infty} \frac{\left(1+\rho^{4 n}-2 \rho^{2 n} \cos 2 \phi\right)}{\left(1+\rho^{4 n}-2 \rho^{2 n} \cos \phi\right)^{4}}>0 .
$$

Therefore, because $\partial L / \partial \phi\left(e^{\mathrm{i} \phi}\right)=\mathrm{i} M\left(e^{\mathrm{i} \phi}\right), L\left(e^{\mathrm{i} \phi}\right)$ decreases when $0<\phi<\pi$, and $L\left(e^{\mathrm{i} \phi}\right)$ increases when $\pi<\phi<2 \pi$. The minimum of $L\left(e^{\mathrm{i} \phi}\right)$ is $L\left(e^{\mathrm{i} \pi}\right)=L(-1)$ where $L(-1)$ can be calculated explicitly to be

$$
L(-1)=\frac{1}{4}+2 \sum_{n=1}^{\infty} \frac{\rho^{2 n}}{\left(1+\rho^{2 n}\right)^{2}}>0 .
$$

Therefore $L\left(e^{\mathrm{i} \phi}\right)$ is positive over this range of $\phi$. 


\section{B Proof of the Properties of $\alpha_{\theta}$ and $\beta_{\theta}$}

Here we will prove that for $0<\theta<\pi, \alpha_{\theta}$ is a monotonically increasing function and $\beta_{\theta}$ is a monotonically decreasing function with respect to $\theta$, that is,

$$
\frac{\partial \alpha_{\theta}}{\partial \theta}>0, \quad \frac{\partial \beta_{\theta}}{\partial \theta}<0 .
$$

We will prove it using the integrated Fay trisecant identity. From (52),

$$
\frac{\partial \alpha_{\theta}}{\partial \theta} X_{0, \pi, \theta}+\frac{\partial \beta_{\theta}}{\partial \theta} Y_{0, \pi, \theta}=0
$$

which means the sign of $\partial \alpha_{\theta} / \partial \theta$ is opposite to $\partial \beta_{\theta} / \partial \theta$. By a log-sum and sum-log inequality,

$$
\begin{aligned}
\log \left[\alpha_{\theta^{\prime}} X_{0, \pi, \theta}\right. & \left.+\beta_{\theta^{\prime}} Y_{0, \pi, \theta}\right] \\
& \geq \frac{1}{2 \pi} \int_{0}^{2 \pi} \log \left[A\left(0, \theta^{\prime}, \rho e^{\mathrm{i} \phi}\right) X_{0, \pi, \theta}+B\left(\pi, \theta^{\prime}, \rho e^{\mathrm{i} \phi}\right) Y_{0, \pi, \theta}\right] d \phi \\
& =\frac{1}{2 \pi} \int_{0}^{2 \pi} \log \left[A\left(0, \theta, \rho e^{\mathrm{i} \phi}\right) X_{0, \pi, \theta}+B\left(\pi, \theta, \rho e^{\mathrm{i} \phi}\right) Y_{0, \pi, \theta}\right] d \phi=0,
\end{aligned}
$$

where we used (90) and (91). This means

$$
\alpha_{\theta^{\prime}} X_{0, \pi, \theta}+\beta_{\theta^{\prime}} Y_{0, \pi, \theta} \geq 1
$$

From the Fay trisecant identity, $\alpha_{\theta} X_{0, \pi, \theta}+\beta_{\theta} Y_{0, \pi, \theta}=1$, so

$$
\left(\alpha_{\theta^{\prime}}-\alpha_{\theta}\right) X_{0, \pi, \theta}+\left(\beta_{\theta^{\prime}}-\beta_{\theta}\right) Y_{0, \pi, \theta} \geq 0
$$

for $0<\theta^{\prime}<\pi$. Hence, together with (102), we can obtain an inequality for the second derivatives of $\alpha_{\theta}$ and $\beta_{\theta}$ as follows:

$$
\frac{\partial^{2} \alpha_{\theta}}{\partial \theta^{2}} X_{0, \pi, \theta}+\frac{\partial^{2} \beta_{\theta}}{\partial \theta^{2}} Y_{0, \pi, \theta} \geq 0
$$

When we take the derivative of (102) with respect to $\theta$, we get

$$
\frac{\partial \alpha_{\theta}}{\partial \theta} \frac{\partial X_{0, \pi, \theta}}{\partial \theta}+\frac{\partial \beta_{\theta}}{\partial \theta} \frac{\partial Y_{0, \pi, \theta}}{\partial \theta}+\frac{\partial^{2} \alpha_{\theta}}{\partial \theta^{2}} X_{0, \pi, \theta}+\frac{\partial^{2} \beta_{\theta}}{\partial \theta^{2}} Y_{0, \pi, \theta}=0 .
$$

Thus, from (106),

$$
\frac{\partial \alpha_{\theta}}{\partial \theta} \frac{\partial X_{0, \pi, \theta}}{\partial \theta}+\frac{\partial \beta_{\theta}}{\partial \theta} \frac{\partial Y_{0, \pi, \theta}}{\partial \theta}=-\frac{\partial^{2} \alpha_{\theta}}{\partial \theta^{2}} X_{0, \pi, \theta}-\frac{\partial^{2} \beta_{\theta}}{\partial \theta^{2}} Y_{0, \pi, \theta} \leq 0 .
$$


The signs of $\partial X_{0, \pi, \theta} / \partial \theta$ and $\partial Y_{0, \pi, \theta} / \partial \theta$ can be determined for $0<\theta<\pi$, that is,

$$
\begin{aligned}
& \frac{\partial X_{0, \pi, \theta}}{\partial \theta}=\mathrm{i} X_{0, \pi, \theta}\left[K\left(-e^{\mathrm{i} \theta}\right)-K\left(-e^{-\mathrm{i} \theta}\right)\right]=\mathrm{i} X_{0, \pi, \theta}\left[2 K\left(-e^{\mathrm{i} \theta}\right)-1\right]<0, \\
& \frac{\partial Y_{0, \pi, \theta}}{\partial \theta}=\mathrm{i} Y_{0, \pi, \theta}\left[K\left(e^{\mathrm{i} \theta}\right)-K\left(e^{-\mathrm{i} \theta}\right)\right]=\mathrm{i} Y_{0, \pi, \theta}\left[2 K\left(e^{\mathrm{i} \theta}\right)-1\right]>0,
\end{aligned}
$$

where we used the positiveness of $L\left(e^{\mathrm{i} \phi}\right)$ to state that the imaginary part of $K\left(e^{\mathrm{i} \phi}\right)$ is monotonically increasing and $K\left(e^{\mathrm{i} \pi}\right)=1 / 2$. Because the sign of $\partial \alpha_{\theta} / \partial \theta$ is opposite to $\partial \beta_{\theta} / \partial \theta$ as deduced from (102), we can conclude that

$$
\frac{\partial \alpha_{\theta}}{\partial \theta}>0, \quad \frac{\partial \beta_{\theta}}{\partial \theta}<0 .
$$

\section{References}

1. Miccoli, I., Edler, F., Pfnür, H., Tegenkamp, C.: 2015 The 100th anniversary of the four-point probe technique: the role of probe geometries in isotropic and anisotropic systems. J. Phys. Condens. Matter 27, 223201

2. Singh, Y.: 2013 Electrical resistivity measurements: a review. In: Int. J. Mod. Phys. Conf. Ser., vol. 22, pp. 745-756. World Scientific

3. Van der Pauw, L.J.: A method of measuring the resistivity and Hall coefficient on lamellae of arbitrary shape. Philips Tech. Rev. 20, 220-224 (1958)

4. Van der Pauw, L.J.: A method of measuring specific resistivity and Hall effect of discs of arbitrary shape. Philips Res. Rep 13, 1-9 (1958)

5. Sun, Y., Shi, J., Meng, Q.: Measurement of sheet resistance of cross microareas using a modified van der Pauw method. Semiconductor Sci. Technol. 11, 805 (1996)

6. Cieśliński, J.L.: Modified van der Pauw method based on formulas solvable by the Banach fixed point method. Thin Solid Films 522, 314-317 (2012)

7. Szymański, K., Cieśliński, J.L., Łapiński, K.: Van der Pauw method on a sample with an isolated hole. Phys. Lett. A 377, 651-654 (2013)

8. Szymański, K., Łapiński, K., Cieśliński, J.L.: Determination of the Riemann modulus and sheet resistance of a sample with a hole by the van der Pauw method. Meas. Sci. Technol. 26, 055003 (2015)

9. Oh, D., Ahn, C., Kim, M., Park, E.K., Kim, Y.S.: Application of the van der Pauw method for samples with holes. Meas. Sci. Technol. 27:125001 (2016)

10. Sun, Z.H., Zhou, J., Xia, X.J., Zhou, D.M.: Two-dimensional electrostatic model for the Van der Pauw method. Phys. Lett. A 381, 2144-2148 (2017)

11. Szymański, K.R., Walczyk, C.J., Cieśliński, J.L.: Determination of topological properties of thin samples by the van der Pauw method. Measurement 145, 568-572 (2019)

12. Szymański, K., Łapiński, K., Cieśliński, J.L., Kobus, A., Zaleski, P., Biernacka, M., Perzyńska, K.: Determination of the Riemann modulus and sheet resistivity by a six-point generalization of the van der Pauw method. Meas. Sci. Technol. 26, 1-9 (2015)

13. Szymański, K., Zaleski, P.: Precise measurement of inhomogeneity of 2-D system by six-point method. IEEE Trans. Instrum. Measurement 66, 1243-1247 (2017)

14. Koon, D., Wang, F., Petersen, D., Hansen, O.: Sensitivity of resistive and Hall measurements to local inhomogeneities: Finite-field, intensity, and area corrections. J. Appl. Phys. 116:133706 (2014)

15. Koon, D., Her̂manová, M., Náhlík, J. : Electrical conductance sensitivity functions for square and circular cloverleaf van der Pauw geometries. Meas. Sci. Technol. 26:115004 (2015)

16. Ausserlechner, U.: The classical Hall effect in multiply-connected plane regions part I: topologies with stream function. J. Appl. Math. Phys. 7, 1968-1996 (2019)

17. Náhlík, J., Kaspárková, I., Fitl, P.: Study of quantitative influence of sample defects on measurements of resistivity of thin films using van der Pauw method. Measurement 44, 1968-1979 (2011) 
18. Crowdy, D.G.: Solving problems in multiply connected domains, CBMS-NSF Regional Conf. Ser. in Appl. Math., SIAM, (2020)

19. Miyoshi, H., Crowdy, D.G., Nelson, R.: Fay meets van der Pauw: the trisecant identity and the resistivity of holey samples. Proc. Roy. Soc. A 477, 20200796 (2021)

20. Goluzin, G.M.: Geometric function theory of a complex variable. Am. Math, Soc. (1969)

21. Nehari, Z.: Conformal Mapping. Courier Corporation (2012)

22. Ablowitz, M.J., Fokas, A.S.: Complex Variables: Introduction and Applications. Cambridge University Press, Cambridge (2003)

23. Crowdy, D.G.: Analytical formulae for source and sink flows in multiply connected domains. Theor. Comp. Fluid Dyn. 27, 1-19 (2013)

24. Fay, J.D.: Theta functions on Riemann surfaces, vol. 352. Springer (2006)

25. Poor, C.: Fay's trisecant formula and cross-ratios. Proc. Am. Math. Soc. 114, 667-671 (1992)

Publisher's Note Springer Nature remains neutral with regard to jurisdictional claims in published maps and institutional affiliations. 